Article

\title{
Synthesis and Biological Evaluation of New Cholinesterase Inhibitors for Alzheimer's Disease
}

\author{
Weiam Hussein 1,2, Begüm Nurpelin Sağlık ${ }^{2,3}$, Serkan Levent ${ }^{2,3}$, Büşra Korkut ${ }^{4}$, Sinem Ilgın ${ }^{4}$, \\ Yusuf Özkay 2,3,* (iD) and Zafer Asım Kaplancıklı 2 iD \\ 1 Department of Pharmaceutical Chemistry, Faculty of Pharmacy, Aden University, 6075 Aden, Yemen; \\ white.wf.rose@gmail.com \\ 2 Department of Pharmaceutical Chemistry, Faculty of Pharmacy, Anadolu University, 26470 Eskişehir, Turkey; \\ bnsaglik@anadolu.edu.tr (B.N.S.); serkanlevent@anadolu.edu.tr (S.L.); zakaplan@anadolu.edu.tr (Z.A.K.) \\ 3 Doping and Narcotic Compounds Analysis Laboratory, Faculty of Pharmacy, Anadolu University, 26470 \\ Eskişehir, Turkey \\ 4 Department of Pharmaceutical Toxicology, Faculty of Pharmacy, Anadolu University, 26470 Eskişehir, \\ Turkey; busrakorkut@anadolu.edu.tr (B.K.); silgin@anadolu.edu.tr (S.I.) \\ * Correspondence: yozkay@anadolu.edu.tr; Tel.: +90-222-335-0580 (ext. 3779)
}

Academic Editor: Praveen P. Nekkar Rao

Received: 29 July 2018; Accepted: 12 August 2018; Published: 14 August 2018

\begin{abstract}
Alzheimer's disease (AD) is a neurodegenerative disorder mostly influencing the elderly, and causes death due to dementia. The main pathogenic feature connected with the progression of this multifactorial disease is the weakening of the cholinergic system in the brain. Cholinesterase (ChE) inhibitors are recognized as one of the choices in the treatment of AD. The inhibition of acetylcholinesterase (AChE) and butyrylcholinesterase (BChE) were approved as a therapeutic strategy to reduce the symptoms of $\mathrm{AD}$ and prevent its progression. The capacity of $\mathrm{BChE}$ is not completely known yet; rather, it is accepted to assume a part in a few disorders such as AD. Thus, $\mathrm{BChE}$ inhibitors may have a greater role for the treatment of $\mathrm{AD}$ in the future. In the present study, 2-(9-acridinylamino)-2-oxoethyl piperazine/piperidine/morpholinecarbodithioate derivatives were synthesized in order to investigate anticholinesterase activity. Eight derivatives demonstrated a specific and promising action against BChE. Furthermore, compound $4 \mathrm{n}$ showed inhibitory activity against both enzymes. It was found that the active compounds were well tolerated in the cytotoxicity test. Possible interactions between the lead compound, $\mathbf{4 n}$, and the BChE enzyme were determined through a docking study. The findings obtained within this paper will contribute to the development of new and effective synthetic anti-Alzheimer compounds, and will ideally encourage future screening against $\mathrm{AD}$.
\end{abstract}

Keywords: Alzheimer's disease; acetylcholinesterase; butyrylcholinesterase; 9-aminoacridine; dithiocarbamate salts; docking study

\section{Introduction}

Alzheimer's disease (AD) is a neurological disorder and is one of the most common causes of dementia in the age group above 60 years, for which there is no radical cure. It is estimated from the world population that more than 35.6 million people are currently living with $\mathrm{AD}$, and this may increase to 65.7 million by 2030, and 115.4 million by 2050 [1]. According to a World Health Organization (WHO) report, more than $50 \%$ of people with AD live in the developing world, and it is estimated that this population will be $70 \%$ by 2025 [2]. To date, some cases of low levels of acetylcholine (ACh), oxidative anxiety, dyshomeostasis of biometals, and amyloid- $\beta(\mathrm{A} \beta)$ stores were exhibited to be related to $\mathrm{AD}$ pathogenesis [3]. In light of these findings, a few speculations were proposed to clarify the 
progress of AD. The most recognized theory includes the loss of cholinergic neurons in the cerebrum of patients with $\mathrm{AD}$, and a reduced action of choline acetyltransferase, which catalyzes the generation of ACh. Thus, cognitive dysfunction occurs and neurotransmission levels decrease [4]. In AD, although AChE is generally the primary medication target, recent investigations were additionally centered on a scan for BChE inhibitors [5-8]. One of the most interesting studies showed that, when BChE progresses toward the formation of A $\beta$ plaques, its advanced role involves the conversion of "harmless" plaques to "damaging" plaques [9]. Scientists additionally discovered a relationship between a less active variant of BChE (Ala539Thr) and a lower inclination toward growing AD [10]. Therefore, BChE represents a proper imaging target for early diagnosis and treatment of AD. In this way, new remedial approaches are presently in progress, and exploratory medicine surrounding this approach sped up during the recent decade.

Carbamates are the most generally contemplated class of AChE inhibitors, on which impressive research was performed in connection to AD. Rivastigmine, a dual AChE and BChE inhibitor, is one of the most broadly utilized anticholinesterase agents bearing a carbamate moiety [11,12]. Dithiocarbamates have a great deal of interest in the design of new compounds, which can be obtained via the bioisosteric substitution of a carbamate moiety with a dithiocarbamate moiety. They are critical pharmacophores due to their lipophilicity, which is essential for the transport of drugs to the central nervous system (CNS) through the blood-brain barrier (BBB) $[13,14]$. Tacrine and donepezil are other anticholinesterase agents that selectively inhibit BChE and $\mathrm{AChE}$, respectively. The use of tacrine is limited as it may cause an increase in the level of serum aminotransferase, which is connected to intense liver harm. On the other hand, donepezil is a more favorable AChE inhibitor since it has some advantages such as non-hepatotoxicity, and the lowest number of side effects when consumed once daily [15]. Thus, several studies on compounds structurally related to tacrine were performed to discover new BChE inhibitors with reduced side effects [16-23]

It was reported that anticholinesterase agents ought to feature a central ring, a basic region, and a suitable connection between the central ring and basic region, such as $\mathrm{O}, \mathrm{CH}_{2}, \mathrm{CONH}_{\text {, }}$ and $\mathrm{CONH}\left(\mathrm{CH}_{2}\right)$ n, to satisfy the main structural necessity for active and strong enzyme inhibitors [15]. In addition, the bulky moieties were reported to possess good anti-BChE activity, which may be related to the ability of BChE to ideally bind with bigger substituents, as its structure is composed of a more open core [24]. Furthermore, there are no reported data on 9-aminoacridine and its acetylated analogs as anticholinesterase inhibitors until this study. Based on this empirical knowledge, we aimed to improve BChE inhibitory activity via the design of new compounds bearing the following structural properties: 9-aminoacridine as the central ring, heterocyclic rings such as piperazine, piperidine, or morpholine as the basic region, and finally, a (thiocarbamoylthio)acetamide moiety as the connection between the central ring and the basic region (Figure 1). Thus, the aim was to synthesize new hybrids of 9-aminoacridine and dithiocarbamates analogs as anticholinesterase agents. 


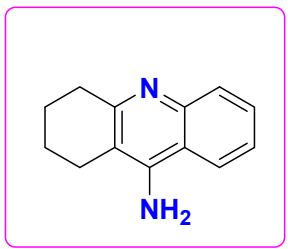

Tacrine

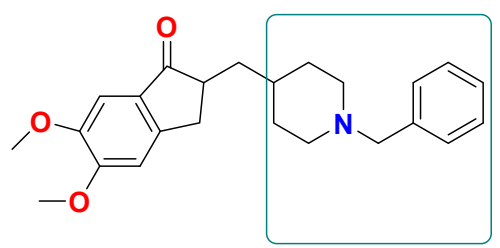

Donepezil

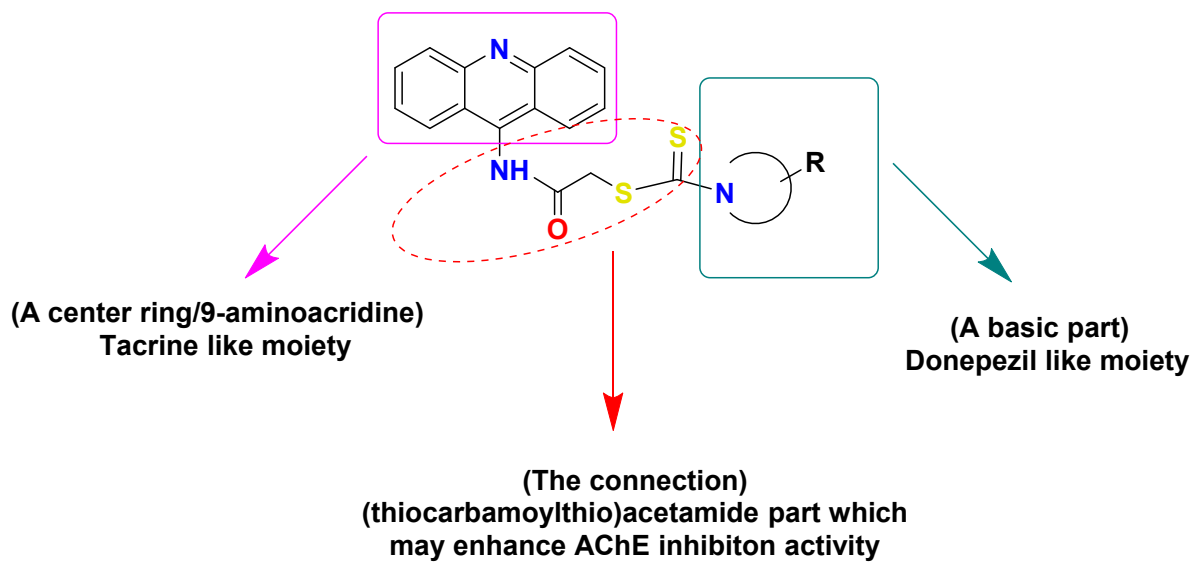

Figure 1. Design strategy of recently synthesized derivatives.

\section{Results and Discussion}

\subsection{Chemistry}

The newly synthesized derivatives (4a-4u) were obtained by taking advantage of the three-step protocol as outlined in Scheme 1. The treatment of $N$-(9-acridinyl)-2-chloroacetamide derivatives (2) with the appropriate sodium $N$-substituted piperazine/piperidine/morpholine dithiocarbamates (3) gave the target compounds with yields of $81.0-97.0 \%$. The structures of all synthesized compounds were elucidated using infrared (IR), high-resolution mass spectrometry (HRMS), ${ }^{1} \mathrm{H}-\mathrm{NMR}$, and ${ }^{13} \mathrm{C}$-NMR spectroscopic methods. All compounds indicated logical, analytical, and spectroscopic information strongly correlating with their structures.

\subsection{Enzymatic Inhibition}

The results of biological assays were evaluated to understand the inhibitory potency of the newly synthesized compounds (4a-4u) against cholinesterase enzymes of different species (AChE from Electrophorus electricus and human cells, and BChE from equine and human cells). In order to acquire effective knowledge about structure activity relationship (SAR), compounds $\mathbf{4 a}-\mathbf{4} \mathbf{u}$ were divided into two classes as derivatives containing piperazine (4a-4q) and morpholine or piperidine (4r-4u). The assay was performed in two steps. Firstly, compounds $4 \mathbf{a}-\mathbf{4 u}$ were tested at $10^{-3}$ and $10^{-4} \mathrm{M}$ concentrations. The second step was performed by using $10^{-5}-10^{-9} \mathrm{M}$ concentrations of selected compounds that indicated more than $50 \%$ inhibitory activity at the initial concentrations. It was clearly observed that similar results were obtained against both species of $\mathrm{AChE}$ and $\mathrm{BChE}$. The reason for this similarity was thought to be high degree of homology in the enzymes of the studied species (Supplementary Materials and Table 1).

Our in-depth analysis showed that the incorporation of a 9-aminoacridine moiety into the designed compounds contributed weakly to the AChE inhibitory activity; however, it strongly increased the inhibitory activity toward BChE (Supplementary Materials and Table 1). 
<smiles>Nc1c2ccccc2nc2ccccc12</smiles>

1

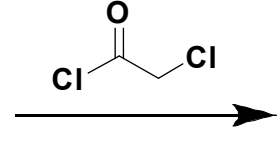

a<smiles>O=C(CCl)Nc1c2ccccc2nc2ccccc12</smiles>

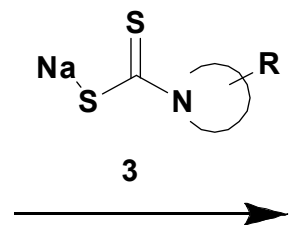

b<smiles>[R]CCCN(CC)C(=S)SCC(=O)Nc1c2ccccc2nc2ccccc12</smiles>

Comp.

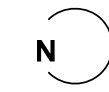

Piperazinyl Piperazinyl Piperazinyl Piperazinyl Piperazinyl Piperazinyl Morpholinyl Piperidinyl Piperidinyl Piperidinyl

\section{$\mathbf{R}$}

4-cyclohexyl 4-benzhydryl 4-[4-(trifluoromethyl)benzyl] 4-(4-methylbenzyl) 4-(4-fluorophenyl) 4-(2-furoyl)

Piperazinyl Piperazinyl Piperazinyl Piperazinyl Piperazinyl Piperazinyl 4-(4-nitrophenyl)
4-(2-hydroxyethyl) 4-phenyl 4-(4-methoxyphenyl) 4-(4-chlorophenyl) 4-(pyrimidin-2-yl) 4-benzyl
$4 \mathrm{~m}$

40

$4 p$

$4 q$

$4 s$

$4 t$
$4 u$ 4-(2-furoyl)

2-methyl 4-benzyl

Scheme 1. Synthesis of compounds $4 a-4 u$. (a): $\left(\mathrm{C}_{2} \mathrm{H}_{5}\right)_{3} \mathrm{~N} / \mathrm{THF} / 0{ }^{\circ} \mathrm{C}$ then $\mathrm{rt}$; (b) acetone/reflux.

Compounds in the first class $(\mathbf{4} \mathbf{a}-\mathbf{4} \mathbf{q})$ offered inhibitory activity with an $\mathrm{IC}_{50}$ ranging from 0.014 to $2.097 \mu \mathrm{M}$ against BChE. Compounds $4 \mathbf{a}, 4 \mathbf{b}, 4 \mathbf{e}, 4 \mathbf{i}, 4 \mathrm{~m}, \mathbf{4 n}$, and $4 \mathbf{o}$ possessing piperazine with 2-dimethylaminoethyl, 3-dimethylaminopropyl, 2-hydroxyethyl, 4-chlorophenyl, 4-benzhydryl, 4-(trifluoromethyl)benzyl, and 4-methylbenzyl respectively, showed more potent BChE inhibitory activity than other derivatives in the series. Furthermore, the most active compound, $4 \mathrm{~m}\left(\mathrm{IC}_{50}\right.$ : $0.092 \mu \mathrm{M})$, exhibited 15.4-fold better BChE inhibition in comparison with the positive control, donepezil $\left(\mathrm{IC}_{50}: 1.419 \mu \mathrm{M}\right)$. In the second class of compounds, the replacement of piperazine with its bioisosters, namely morpholine (4r) and piperidine (4s, $4 \mathbf{t}$, and $4 \mathbf{u})$, did not significantly enhance the activity against either AChE or BChE.

In terms of SAR, it can be concluded that the variation of secondary amines such as piperazine, piperidine, and morpholine does not affect the enzyme inhibitory potency of the final compounds. However, the substituents on these cyclic amines are mainly responsible for the biological activity. Although the structures of the compounds show great similarities, the presence of trifluoromethyl in compound $4 \mathrm{n}$ significantly influenced the activity. The better inhibitory profile of $4 \mathrm{n}$ than aminoacridine (Table 1) also supports this finding. Thus, it can be suggested that the trifluoromethyl group possesses the ability to form interactions in the enzyme's active site. 
Table 1. Inhibitory activity (\%) of compounds $\mathbf{4 a}-\mathbf{4 u}$ against acetylcholinesterase (AChE) and butyrylcholinesterase (BChE) enzymes at $10^{-4} \mathrm{M}$ concentration and their half maximal inhibitory concentration $\left(\mathrm{IC}_{50} ; \mu \mathrm{M}\right)$ values against these enzymes.

\begin{tabular}{|c|c|c|c|c|c|c|c|c|}
\hline \multirow[b]{2}{*}{ Compound } & \multicolumn{2}{|c|}{$\begin{array}{c}\text { AChE (Electrophorus electricus) } \\
(\%) \pm \mathrm{SD}\end{array}$} & \multicolumn{2}{|c|}{$\begin{array}{l}\text { AChE (Human) } \\
(\%) \pm \text { SD }\end{array}$} & \multicolumn{2}{|c|}{$\begin{array}{l}\text { BChE (Equine) } \\
\quad(\%) \pm \text { SD }\end{array}$} & \multicolumn{2}{|c|}{$\begin{array}{l}\text { BChE (Human) } \\
(\%) \pm \text { SD }\end{array}$} \\
\hline & $10^{-4} \mathrm{M}$ & $\mathrm{IC}_{50}(\mu \mathrm{M})$ & $10^{-4} \mathrm{M}$ & $\mathrm{IC}_{50}(\mu \mathrm{M})$ & $10^{-4} \mathrm{M}$ & $\mathrm{IC}_{50}(\mu \mathrm{M})$ & $10^{-4} \mathrm{M}$ & $\mathrm{IC}_{50}(\mu \mathrm{M})$ \\
\hline $4 a$ & $17.03 \pm 0.48$ & - & $18.15 \pm 0.51$ & - & $* 91.13 \pm 2.94$ & $0.226 \pm 0.008$ & $* 93.25 \pm 2.24$ & $0.243 \pm 0.009$ \\
\hline $4 \mathrm{~b}$ & $32.41 \pm 1.01$ & - & $35.49 \pm 1.00$ & - & $* 82.50 \pm 2.07$ & $0.264 \pm 0.012$ & $* 84.18 \pm 2.21$ & $0.222 \pm 0.010$ \\
\hline $4 c$ & $5.11 \pm 0.09$ & - & $10.28 \pm 0.22$ & - & $22.70 \pm 0.70$ & - & $20.55 \pm 0.61$ & - \\
\hline $4 d$ & $31.64 \pm 0.45$ & - & $34.71 \pm 0.77$ & - & $40.19 \pm 0.49$ & - & $45.26 \pm 0.60$ & - \\
\hline $4 e$ & $7.09 \pm 0.64$ & - & $10.13 \pm 0.51$ & - & $* 95.98 \pm 2.88$ & $1.420 \pm 0.068$ & $* 96.57 \pm 2.03$ & $1.373 \pm 0.057$ \\
\hline $4 f$ & $4.70 \pm 0.27$ & - & $8.71 \pm 0.62$ & - & $12.80 \pm 0.87$ & - & $15.67 \pm 0.79$ & - \\
\hline $4 \mathrm{~g}$ & $10.44 \pm 0.66$ & - & $11.30 \pm 0.58$ & - & $33.65 \pm 0.89$ & - & $35.97 \pm 0.80$ & - \\
\hline $4 \mathrm{~h}$ & $15.23 \pm 0.69$ & - & $18.39 \pm 0.57$ & - & $12.64 \pm 0.46$ & - & $15.58 \pm 0.67$ & - \\
\hline $4 i$ & $35.91 \pm 1.57$ & - & $33.85 \pm 1.15$ & - & $* 69.51 \pm 1.16$ & $2.038 \pm 0.068$ & * $72.90 \pm 1.08$ & $2.097 \pm 0.085$ \\
\hline $4 j$ & $2.88 \pm 0.18$ & - & $5.97 \pm 0.22$ & - & $27.86 \pm 1.07$ & - & $30.77 \pm 1.20$ & - \\
\hline $4 \mathrm{k}$ & $8.62 \pm 0.22$ & - & $7.21 \pm 0.18$ & - & $25.14 \pm 1.42$ & - & $20.65 \pm 1.20$ & - \\
\hline 41 & $3.90 \pm 0.09$ & - & $7.29 \pm 0.18$ & - & $45.88 \pm 1.62$ & - & $42.60 \pm 1.53$ & - \\
\hline $4 \mathrm{~m}$ & $25.43 \pm 1.21$ & - & $27.38 \pm 1.10$ & - & $* 98.96 \pm 3.47$ & $0.092 \pm 0.001$ & $* 97.50 \pm 2.06$ & $0.093 \pm 0.002$ \\
\hline $4 n$ & $* 63.21 \pm 1.76$ & $45.945 \pm 1.581$ & $* 68.39 \pm 1.99$ & $41.129 \pm 1.694$ & $* 99.03 \pm 3.26$ & $0.015 \pm 0.0006$ & $* 98.25 \pm 2.63$ & $0.014 \pm 0.0005$ \\
\hline 40 & $25.30 \pm 1.01$ & - & $30.45 \pm 1.15$ & - & $* 74.83 \pm 2.17$ & $1.071 \pm 0.051$ & $* 75.25 \pm 2.08$ & $1.069 \pm 0.039$ \\
\hline $4 p$ & $48.40 \pm 1.18$ & - & $49.51 \pm 1.50$ & - & $48.65 \pm 1.14$ & - & $40.71 \pm 1.08$ & - \\
\hline $4 q$ & $16.35 \pm 0.39$ & - & $20.28 \pm 0.51$ & - & $39.90 \pm 1.36$ & - & $42.48 \pm 1.22$ & - \\
\hline $4 r$ & $37.33 \pm 1.03$ & - & $40.70 \pm 1.00$ & - & $45.30 \pm 1.88$ & - & $43.29 \pm 1.17$ & - \\
\hline $4 \mathrm{~s}$ & $32.17 \pm 1.23$ & - & $35.20 \pm 1.12$ & - & $38.40 \pm 0.74$ & - & $34.99 \pm 0.63$ & - \\
\hline $4 \mathrm{t}$ & $15.94 \pm 0.42$ & - & $14.51 \pm 0.44$ & - & $* 55.00 \pm 1.74$ & $1.515 \pm 0.072$ & $* 54.26 \pm 1.27$ & $1.607 \pm 0.062$ \\
\hline $4 u$ & $25.88 \pm 0.74$ & - & $30.71 \pm 0.52$ & - & $29.50 \pm 0.48$ & - & $31.21 \pm 0.50$ & - \\
\hline Aminoacridine & $75.18 \pm 1.15$ & $1.752 \pm 0.072$ & $78.22 \pm 0.89$ & $1.339 \pm 0.047$ & $87.29 \pm 1.07$ & $0.233 \pm 0.009$ & $86.12 \pm 1.22$ & $0.221 \pm 0.007$ \\
\hline Donepezil & $98.56 \pm 2.87$ & $0.0077 \pm 0.0003$ & $96.95 \pm 2.14$ & $0.0072 \pm 0.0002$ & $71.65 \pm 2.16$ & $1.683 \pm 0.064$ & $76.50 \pm 2.08$ & $1.419 \pm 0.047$ \\
\hline Tacrine & $97.29 \pm 3.24$ & $0.147 \pm 0.004$ & $98.79 \pm 2.63$ & $0.151 \pm 0.002$ & $98.61 \pm 3.71$ & $0.0068 \pm 0.0002$ & $99.15 \pm 2.07$ & $0.0070 \pm 0.0002$ \\
\hline
\end{tabular}

* Represents the selected compounds for second step due to more than $50 \%$ inhibitory potency at $10^{-4} \mathrm{M}$ concentration. 
It was reported that $\mathrm{BChE}$ contains residues which permit bulkier substrates to enter into the active site [25]. Due to the presence of an acridine ring, the synthesized compounds are big molecules; thus, they may have a higher tendency to inhibit BChE compared to AchE. This situation is also reflected in Table 1 . At $10^{-4} \mathrm{M}$ concentration, all compounds possessed higher inhibition against $\mathrm{BChE}$, indicating that the synthesized compounds had more selectivity toward BChE than AcHE.

\subsection{Kinetics Characterization of BChE Inhibition}

Recognizing the types of enzyme inhibitors is vital; however, much of the drug discovery process focuses instead upon deciding whether one inhibitor is more powerful than another. The two most basic values for evaluating the inhibitor's power and characteristics are $\mathrm{IC}_{50}$ and $\mathrm{K}_{\mathrm{i}}$ [26].

To recognize the inhibition type, enzyme kinetics studies were applied using Lineweaver-Burk plots [27]. For this purpose, we selected compound $4 n$, which showed the best activity against human BChE. There are four different points on the graphs showing the presence (at concentrations of $\mathrm{IC}_{50} / 2, \mathrm{IC}_{50}$, and $2 \times \mathrm{IC}_{50}$ ) and absence (control) of $4 \mathbf{n}$. As seen in Figure 2, every single straight line intersected in the second quadrant of the coordinate axis, which characterizes a typical mixed inhibition. In addition, a graphical examination of the corresponding Lineweaver-Burk plot shows both increased slopes (decreased $\mathrm{V}_{\max }$ ) and intercepts (higher $\mathrm{K}_{\mathrm{m}}$ ) at higher inhibitor concentration. This pattern also indicates a mixed-type inhibition.

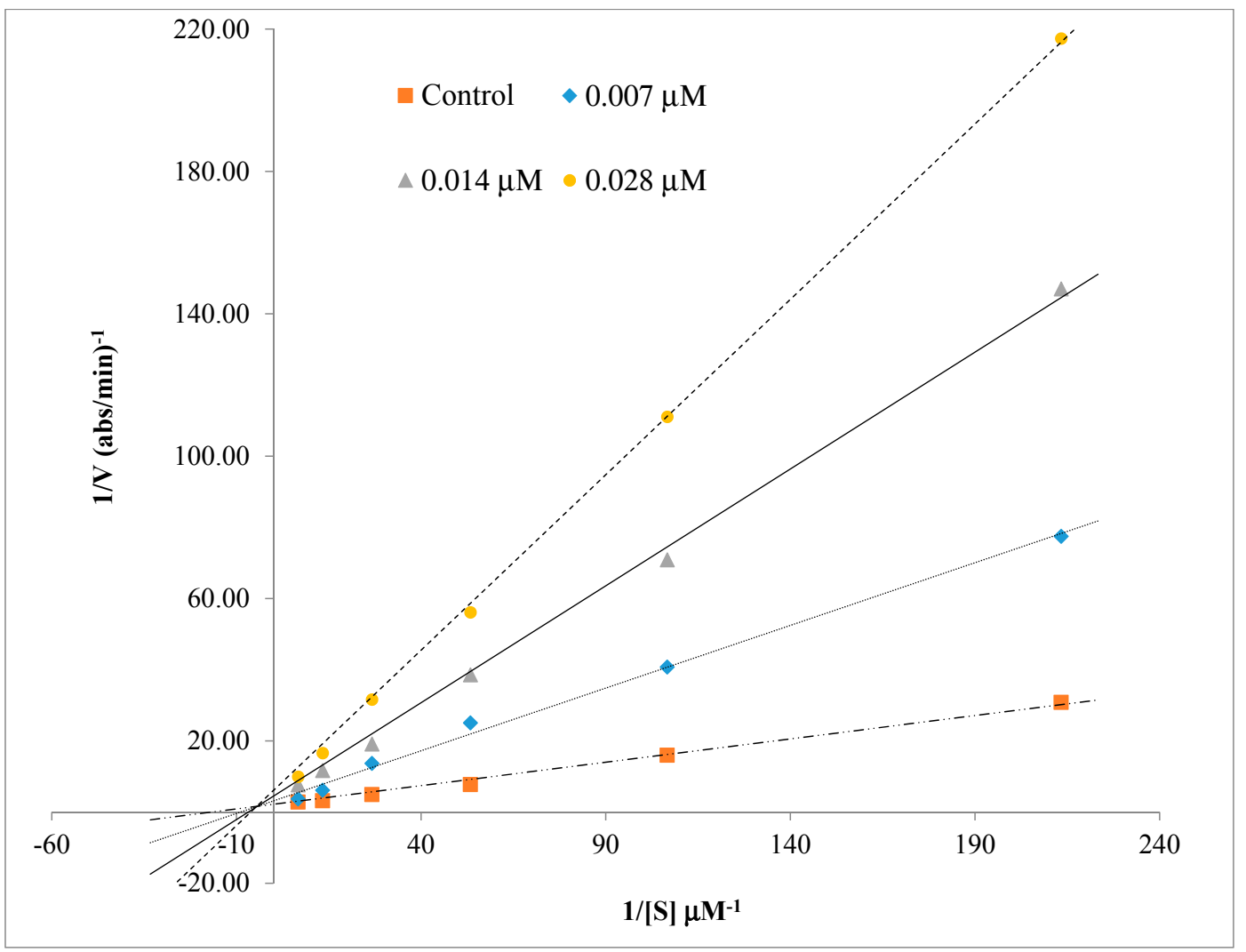

Figure 2. Lineweaver-Burk plot for the inhibition of human butyrylcholinesterase (BChE) by compound $4 \mathrm{n}$ at different concentrations of substrate butyrylthiocholine iodide (BTCI).

The calculation of the inhibition constant $\left(\mathrm{K}_{\mathrm{i}}\right)$ is an effective tool for measuring the affinity of an enzyme for its substrate, and it is easily determined using the secondary plots of the Lineweaver-Burk equation. Hence, a graphical investigation for the lead compound, $4 \mathbf{n}$, was performed and is presented in Figure 3. It was determined that $4 \mathrm{n}$ has a very close $\mathrm{K}_{\mathrm{i}}$ value $(0.017 \mu \mathrm{M})$ to that of tacrine [28], meaning that it is able to firmly bind to the $\mathrm{BChE}$ active site. 
When the small molecule binds to the BChE catalytic active site (CAS), the type of inhibition of the enzyme is classified as competitive; by contrast, when the small-molecule interacts with the peripheral anionic site (PAS), the enzymatic inhibition is called non-competitive. Furthermore, once the active molecule acts on both CAS and PAS, the enzymatic inhibition is designated as mixed inhibition [29]. In this manner, the enzyme kinetics study suggests that compound $4 \mathbf{n}$ interacts with both BChE functional sites, CAS and PAS [30].

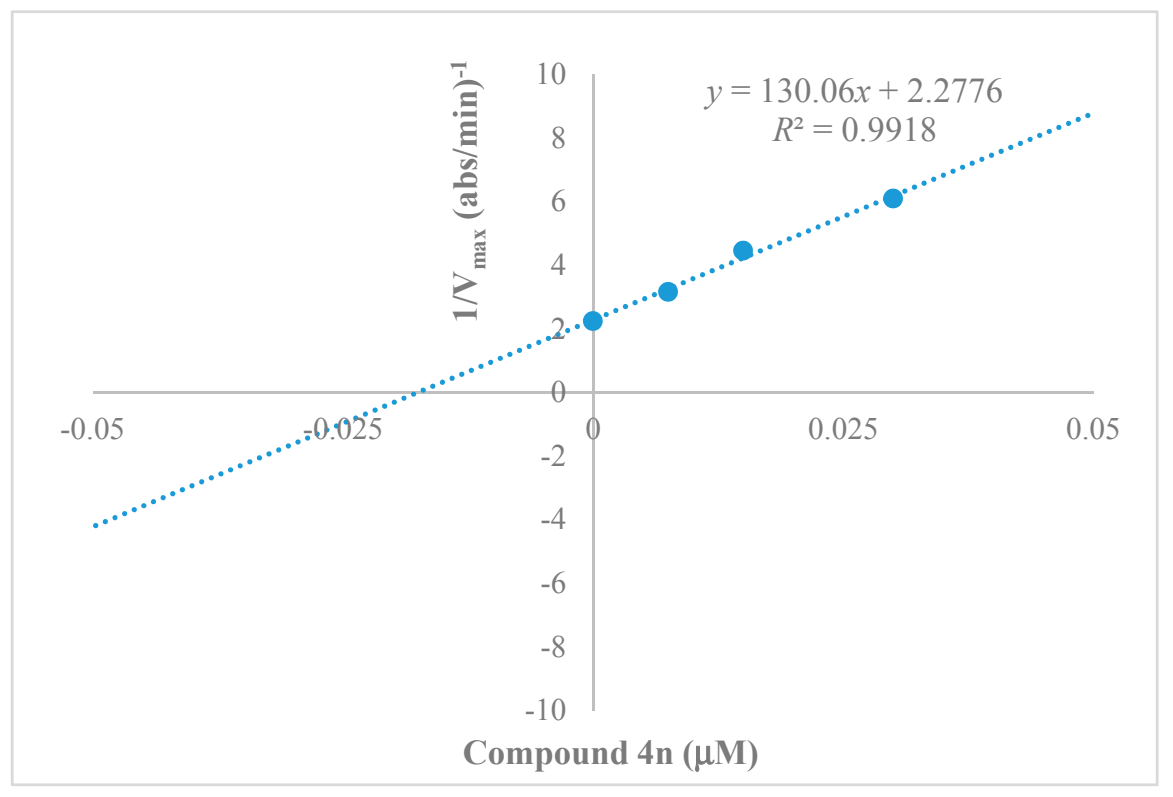

Figure 3. Secondary plot for calculation of the steady-state inhibition constant $\left(\mathrm{K}_{\mathrm{i}}=0.0175 \mu \mathrm{M}\right.$ against BChE) of compound $4 n$.

\subsection{Molecular Docking}

So as to explore a possible interacting mode of the lead compound, $4 \mathbf{n}$, and to evaluate the effects of structural modifications on the BChE enzyme activity, molecular docking was performed and carried out using the X-ray crystal structure of human BChE in complex with tacrine (Protein Data Bank identifier (PDB ID): 4BDS) [31]. Firstly, tacrine was docked in the enzyme's active site to validate the docking procedure. Thus, the $\pi-\pi$ interaction between the aromatic ring of tacrine and the indole of Trp82, and the hydrogen bond between the 9-amino group of tacrine and the carbonyl of His 438 were established, as reported in the literature [31].

The docking poses of compound 4n, showing two- and three-dimensional interactions, are presented in Figures 4 and 5. According to the poses, there are three main patterns of interaction. The first one is a $\pi-\pi$ interaction between the phenyl moiety and the imidazole of His438 in the catalytic site of the BChE enzyme, almost identical to that of tacrine. The second pattern involves a hydrogen bond between the amide carbonyl and the amino group of Thr120. The last and probably the most important interaction is another hydrogen bond between the three-fluoro methyl substituent of $4 \mathrm{n}$ and the amino group of Gly115. These results demonstrate that $\mathbf{4 n}$ has a significant potency to strongly bind to BChE. In particular, the last interaction clarifies why compound $4 \mathrm{n}$ is more active than other derivatives in the series. Findings of the docking analyses confirmed the data reporting that the bulky moieties have good anti-BChE activity due to their binding ability in the active site of BChE [24,25]. 


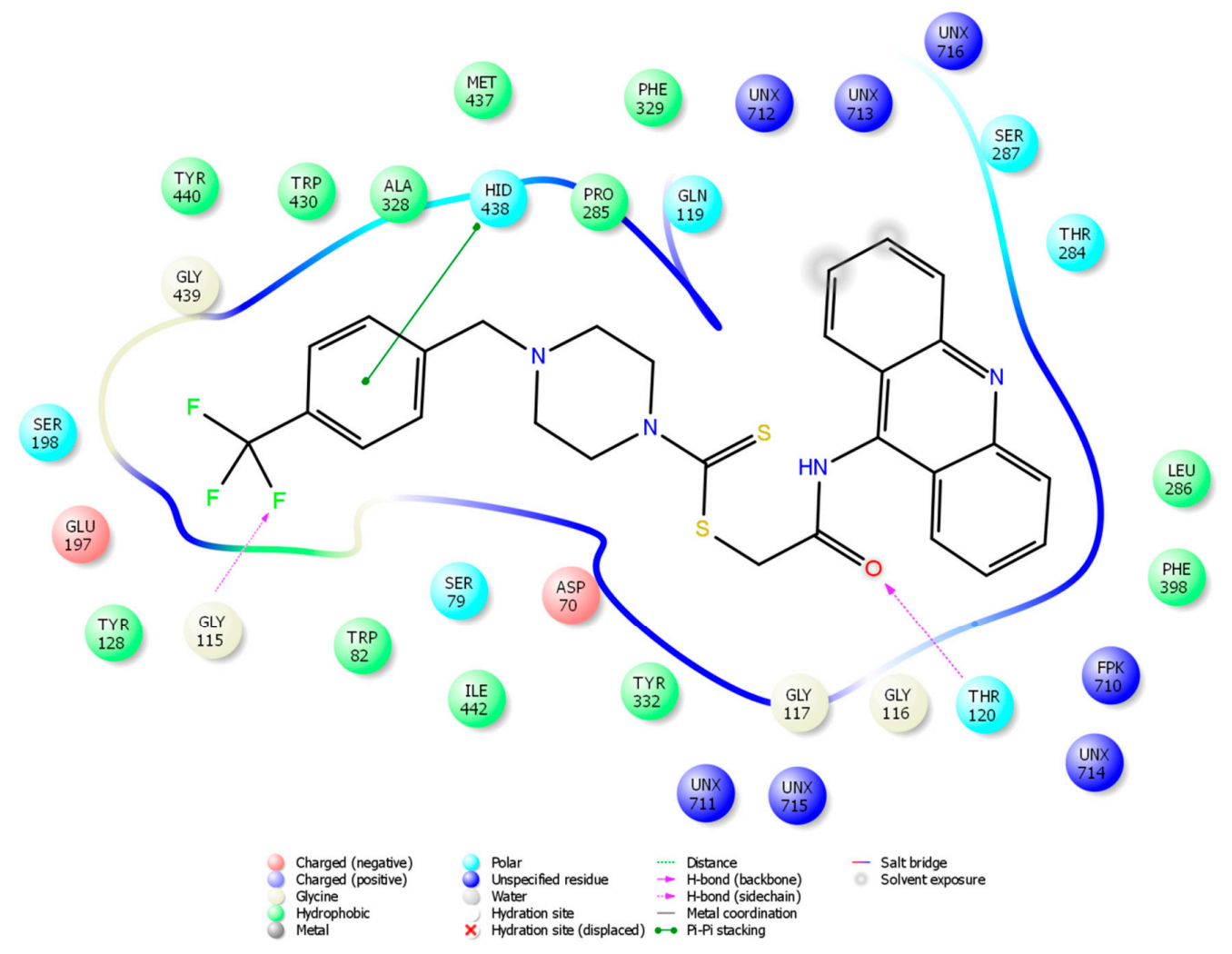

Figure 4. Two-dimensional interaction of compound 4n with BChE.

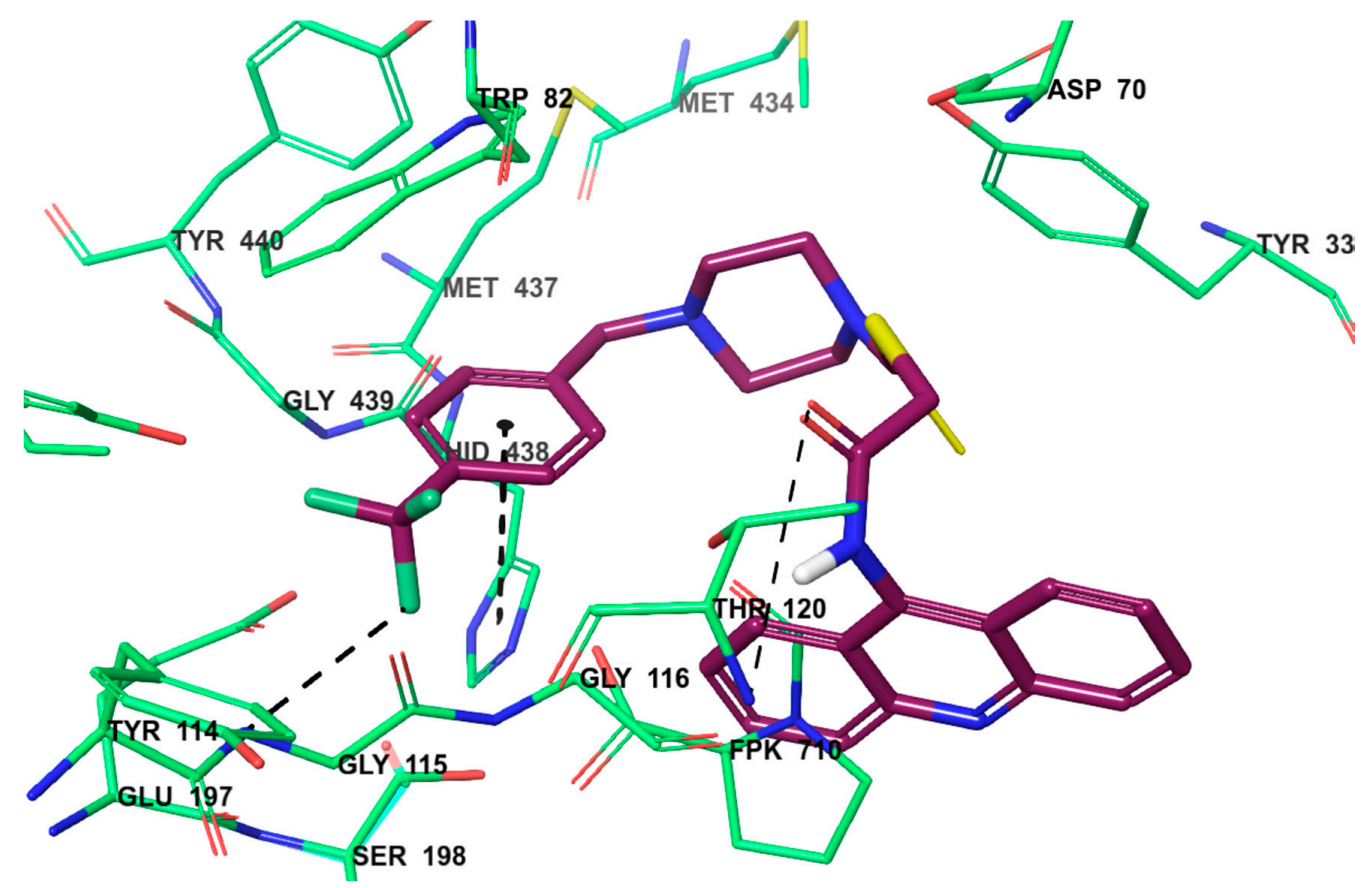

Figure 5. Three-dimensional interaction of compound $4 \mathrm{n}$ with BChE.

\subsection{MTT Cell Viability Assay and Selectivity Indexes}

The active compounds $(\mathbf{4 a}, \mathbf{4 b}, \mathbf{4 e}, \mathbf{4 i}, \mathbf{4 m}, \mathbf{4 n}, \mathbf{4 o}$, and $\mathbf{4 t})$ were chosen to determine their cytotoxic activity toward a murine fibroblast healthy cell line (NIH3T3). After incubating the cells with active compounds at concentrations of $0.000316 \mu \mathrm{M}$ to $1000 \mu \mathrm{M}$ for $24 \mathrm{~h}$, the cell viability was measured using 
the 3-(4,5-dimethylthiazol-2-yl)-2,5-diphenyltetrazolium (MTT) assay [15]. The inhibition percentage was calculated for each concentration of the test compounds. The $\mathrm{IC}_{50}$ values of the compounds were determined using a non-linear regression analysis over the calculated percentage inhibition values, and they are presented in Table 2. Accordingly, the cytotoxicity test proposed that the active derivatives were nontoxic against NIH3T3 cells at their active concentrations against BChE.

Table 2. $\mathrm{IC}_{50}$ of selected derivatives against a murine fibroblast healthy cell line (NIH3T3).

\begin{tabular}{cc}
\hline Compound & $\mathrm{IC}_{50}(\mu \mathrm{M})$ \\
\hline $\mathbf{4 a}$ & 26.81 \\
\hline $4 \mathbf{b}$ & 25.64 \\
\hline $4 \mathrm{e}$ & 324.90 \\
\hline $4 \mathbf{i}$ & $>1000$ \\
\hline $4 \mathrm{~m}$ & 214.18 \\
\hline $4 \mathbf{n}$ & $>1000$ \\
\hline $4 \mathbf{o}$ & $>1000$ \\
\hline
\end{tabular}

\subsection{BBB Permeability and Drug-Likeness Score (DLS)}

BBB permeability is exceptionally fundamental for medications that particularly target and focus on the CNS. The failure of medication particles to penetrate the BBB constitutes a major obstacle for CNS drug candidates and ought to be considered in new drug discovery efforts. For this reason, the BBB permeability of the most active derivatives was computed by a CBLigand-BBB forecast server. This predictor uses two different algorithms, AdaBoost and a support vector machine (SVM), combining with four different fingerprints, employed to predict if a compound can pass $(+)$ or cannot pass (-) the BBB. As presented in Table 3, all calculations for the selected compounds resulted in BBB permeability (+), which is necessary for compounds planning to act as ChE inhibitors [32]. The drug-likeness scores (DLS) were additionally calculated for all active compounds using Molsoft's chemical fingerprint mode consisting of 5000 marketed drugs from the World Drug Index (positives) and 10,000 carefully selected non-drug compounds (negatives) [33] According to this program, the DLS scores were observed between 1.26 and 2.21, which establishes and fortifies their therapeutic significance in future studies.

Table 3. Drug-likeness score (DLS) and blood-brain barrier (BBB) permeability of the active compounds.

\begin{tabular}{ccc}
\hline Comp. & DLS & BBB Permeability \\
\hline $\mathbf{4 a}$ & 2.21 & + \\
\hline $\mathbf{4 b}$ & 2.16 & + \\
\hline $\mathbf{4} \mathbf{e}$ & 1.73 & + \\
\hline $\mathbf{4 i}$ & 1.80 & + \\
\hline $\mathbf{4} \mathbf{m}$ & 1.88 & + \\
\hline $\mathbf{4 n}$ & 1.40 & + \\
\hline $\mathbf{4 o}$ & 1.54 & + \\
\hline Tacrine & 0.97 & + \\
\hline Donepezil & 0.91 & + \\
\hline
\end{tabular}




\section{Materials and Methods}

\subsection{Chemistry}

All chemicals were purchased from Sigma-Aldrich Chemicals (Sigma-Aldrich Corp., St. Louis, MO, USA) or Merck Chemicals (Merck KGaA, Darmstadt, Germany). Melting points of the synthesized compounds were assessed with an MP90 digital melting point apparatus (Mettler Toledo, Columbus, $\mathrm{OH}, \mathrm{USA})$, and were uncorrected. The ${ }^{1} \mathrm{H}-\mathrm{NMR}$ and ${ }^{13} \mathrm{C}-\mathrm{NMR}$ spectra were recorded on a Bruker Fourier 300 (Bruker Bioscience, Billerica, MA, USA) in DMSO- $d_{6}$. The IR spectra were obtained on a Shimadzu, IR Affinity 1S (Shimadzu, Kyoto, Japan). The mass spectra of the compounds were taken in negative and positive mode using electron spray ionization (ESI) in LCMS/IT/TOF (Shimadzu, Kyoto, Japan) from the solutions of the samples in methanol. The purities of compounds were checked using thin-layer chromatography (TLC) on silica gel 60 F254 (Merck KGaA, Darmstadt, Germany).

\subsubsection{General Procedure for the Synthesis of N-(9-Acridinyl)-2-Chloroacetamide Derivatives (2)}

Chloroacetyl chloride (30 mmol, $2.4 \mathrm{~mL})$ in THF $(10 \mathrm{~mL})$ was added dropwise while stirring to a mixture of 9-aminoacridine (1; $20 \mathrm{mmol}, 3.9 \mathrm{~g})$ and triethylamine (40 mmol, $5.6 \mathrm{~mL})$ in THF (50 mL) at $0-5{ }^{\circ} \mathrm{C}$ and stirred for $4 \mathrm{~h}$. The solvent was evaporated under reduced pressure. The residue was washed with water to remove triethylamine hydrochloride, before being dried and crystallized from ethanol [34].

3.1.2. General Procedure for the Synthesis of Sodium N-Substituted Piperazine/Morpholine/Piperidine Dithiocarbamates (3a-3u)

An ethanolic solution $(10 \mathrm{~mL})$ of sodium hydroxide $(10 \mathrm{mmol}, 0.4 \mathrm{~g})$ was added to an ethanolic solution $(10 \mathrm{~mL})$ of the secondary amine $(10 \mathrm{mmol})$. The mixture was cooled in an ice bath; additionally, carbon disulfide (100 mmol, $6.0 \mathrm{~mL}$ ) was added dropwise with continuous stirring for $1 \mathrm{~h}$ at room temperature. The precipitates were filtrated and washed with diethyl ether to obtain a white to pale-yellow-colored product in 70-90\% yield [14].

3.1.3. General Procedure for the Synthesis of 2-(9-Acridinylamino)-2-Oxoethyl 4-Piperazinyl/Morpholinyl/Piperidinylcarbodithioate Derivatives (4a-4u)

An equimolar quantity ( $5 \mathrm{mmol}$ ) of appropriate sodium $N, N$-disubstituted dithiocarbamate (3) and of $\mathrm{N}$-(9-acridinyl)-2-chloroacetamide (2) in acetone $(10 \mathrm{~mL})$ were refluxed for $8 \mathrm{~h}$. The mixture was cooled, and the precipitate was filtrated. The residue was washed with water, before being dried and crystallized from ethanol to obtain the final products [14].

2-(9-Acridinylamino)-2-oxoethyl 4-(2-(dimethylamino)ethyl)piperazine-1-carbodithioate (4a). Yield: 88.5\%; melting poing (m.p.): $200.6{ }^{\circ} \mathrm{C}$; FTIR (ATR, $\mathrm{cm}^{-1}$ ): 3269 (amide N-H), 2769-2972 (aliphatic C-H), 1662 $(\mathrm{C}=\mathrm{O}), 1463-1506(\mathrm{C}=\mathrm{N}$ and $\mathrm{C}=\mathrm{C}), 1232(\mathrm{C}=\mathrm{S}), 756$ (out-of-plane $\mathrm{C}-\mathrm{H}$ bending); ${ }^{1} \mathrm{H}-\mathrm{NMR}(300 \mathrm{MHz}$, DMSO-d $\left.d_{6} ; \delta, \mathrm{ppm}\right): 2.17-2.18\left(6 \mathrm{H}, \mathrm{d}, J=2.4 \mathrm{~Hz}, \mathrm{~N}\left(\underline{\mathrm{CH}_{3}}\right)_{2}\right), 2.43-2.44\left(4 \mathrm{H}, \mathrm{t}, J=2.5 \mathrm{~Hz}, \underline{\mathrm{CH}_{2}}-\underline{\mathrm{CH}_{2}}\right)$, 2.48-2.55 $\left(4 \mathrm{H}, \mathrm{m}\right.$, piperazine $\left.\mathrm{C}_{3,5}-\mathrm{H}\right), 3.97$ and $4.25\left(4 \mathrm{H}\right.$, two bs, piperazine $\left.\mathrm{C}_{2,6}-\mathrm{H}\right), 4.62(2 \mathrm{H}, \mathrm{s}$, $\left.\mathrm{COCH}_{2}\right), 7.57-7.62(2 \mathrm{H}, \mathrm{t}, J=7.5 \mathrm{~Hz}, \mathrm{Ar}-\mathrm{H}), 7.82-7.87(2 \mathrm{H}, \mathrm{t}, J=7.7 \mathrm{~Hz}, \mathrm{Ar}-\mathrm{H}), 8.14-8.17(2 \mathrm{H}, \mathrm{d}, J=8.7$ $\mathrm{Hz}, \mathrm{Ar}-\mathrm{H}), 8.25-8.28(2 \mathrm{H}, \mathrm{d}, J=8.6 \mathrm{~Hz}, \mathrm{Ar}-\mathrm{H}), 11.06(1 \mathrm{H}, \mathrm{s},-\mathrm{NH}-)$; ${ }^{13} \mathrm{C}-\mathrm{NMR}\left(75 \mathrm{MHz}, \mathrm{DMSO}-d_{6}\right.$; $\delta$, ppm): $45.7\left(\mathrm{CH}_{3}\right), 50.4\left(\mathrm{CH}_{2}\right), 51.6\left(\mathrm{CH}_{2}\right), 52.9\left(\mathrm{CH}_{2}\right), 55.2\left(\mathrm{CH}_{2}\right), 56.8\left(\mathrm{CH}_{2}\right), 123.2(\mathrm{C}), 125.4(\mathrm{CH})$, $126.2(\mathrm{CH}), 129.6(\mathrm{CH}), 130.9(\mathrm{CH}), 140.6(\mathrm{C}), 149.4$ (C-9 in 9-aminoacridine), $167.4(\mathrm{C}=\mathrm{O}), 194.9(\mathrm{C}=\mathrm{S})$; HRMS $(m / z):[\mathrm{M}+\mathrm{H}]^{+}$calculated for $\mathrm{C}_{24} \mathrm{H}_{29} \mathrm{~N}_{5} \mathrm{OS}_{2}: 468.1886$, found 468.1874 .

2-(9-Acridinylamino)-2-oxoethyl 4-(3-(dimethylamino)propyl)piperazine-1-carbodithioate (4b). Yield: 85.7\%; m.p.: $153.8^{\circ} \mathrm{C}$; FTIR (ATR, $\mathrm{cm}^{-1}$ ): 3340 (amide N-H), 2777-2947 (aliphatic C-H), 1653 (C=O), 1411-1458 $\left(\mathrm{C}=\mathrm{N}\right.$ and $\mathrm{C}=\mathrm{C}$ ), $1209-1263(\mathrm{C}=\mathrm{S}), 758$ (out-of-plane $\mathrm{C}-\mathrm{H}$ bending); ${ }^{1} \mathrm{H}-\mathrm{NMR}\left(300 \mathrm{MHz}, \mathrm{DMSO}-d_{6}\right.$; $\delta, \mathrm{ppm}): 1.47-1.56\left(2 \mathrm{H}, \mathrm{m}, \mathrm{CH}_{2}-\mathrm{CH}_{2}-\mathrm{CH}_{2}\right), 2.08\left(6 \mathrm{H}, \mathrm{s}, \mathrm{N}\left(\underline{\mathrm{CH}_{3}}\right)_{2}\right), 2.15-2.20(2 \mathrm{H}, \mathrm{t}, J=7.1 \mathrm{~Hz}$, 
$\left.\mathrm{CH}_{2}-\mathrm{CH}_{2} \mathrm{CH}_{2} \mathrm{~N}\left(\mathrm{CH}_{3}\right)_{2}\right), 2.24-2.29\left(2 \mathrm{H}, \mathrm{d}, J=7.4 \mathrm{~Hz}, \mathrm{CH}_{2} \mathrm{CH}_{2} \mathrm{CH}_{2}\right), 2.41-2.42(4 \mathrm{H}, \mathrm{d}, J=0.9$, piperazine $\left.\mathrm{C}_{3,5}-\mathrm{H}\right), 3.98$ and $4.26\left(4 \mathrm{H}\right.$, two s, piperazine $\left.\mathrm{C}_{2,6}-\mathrm{H}\right), 4.65\left(2 \mathrm{H}, \mathrm{s}, \mathrm{COCH}_{2}\right), 7.57-7.62(2 \mathrm{H}, \mathrm{q}, J=7.6 \mathrm{~Hz}$, $\mathrm{Ar}-\mathrm{H}), 7.81-7.87(2 \mathrm{H}, \mathrm{m}, \mathrm{Ar}-\mathrm{H}), 8.14-8.17(2 \mathrm{H}, \mathrm{d}, J=8.7 \mathrm{~Hz}, \mathrm{Ar}-\mathrm{H}), 8.26-8.29(2 \mathrm{H}, \mathrm{d}, J=8.7 \mathrm{~Hz}, \mathrm{Ar}-\mathrm{H})$, $11.19(1 \mathrm{H}, \mathrm{s},-\mathrm{NH}-) ;{ }^{13} \mathrm{C}-\mathrm{NMR}\left(75 \mathrm{MHz}, \mathrm{DMSO}-d_{6} ; \delta, \mathrm{ppm}\right): 24.9\left(\mathrm{CH}_{2}\right), 45.7\left(\mathrm{CH}_{2}\right), 50.4\left(\mathrm{CH}_{3}\right), 51.6$ $\left(\mathrm{CH}_{2}\right), 52.9\left(\mathrm{CH}_{2}\right), 55.2\left(\mathrm{CH}_{2}\right), 56.7\left(\mathrm{CH}_{2}\right), 123.2(\mathrm{C}), 125.4(\mathrm{CH}), 126.2(\mathrm{CH}), 129.6(\mathrm{CH}), 130.9(\mathrm{CH})$, 140.6 (C), 149.4 (C-9 in 9-aminoacridine), $167.4(\mathrm{C}=\mathrm{O}), 194.9$ (C=S); HRMS ( $m / z)$ : $[\mathrm{M}+\mathrm{H}]^{+}$calculated for $\mathrm{C}_{25} \mathrm{H}_{31} \mathrm{~N}_{5} \mathrm{OS}_{2}$ : 482.2043, found 482.2047.

2-(9-Acridinylamino)-2-oxoethyl 4-ethylpiperazine-1-carbodithioate (4c). Yield: $81.0 \%$; m.p.: $221.7{ }^{\circ} \mathrm{C}$; FTIR (ATR, $\mathrm{cm}^{-1}$ ): 3232 (amide N-H), 2358-2974 (aliphatic $\left.\mathrm{C}-\mathrm{H}\right), 1654(\mathrm{C}=\mathrm{O}), 1435-1508(\mathrm{C}=\mathrm{N}$ and $\mathrm{C}=\mathrm{C}), 1219-1271(\mathrm{C}=\mathrm{S}), 732-758$ (out-of-plane $\mathrm{C}-\mathrm{H}$ bending); ${ }^{1} \mathrm{H}-\mathrm{NMR}\left(300 \mathrm{MHz}\right.$, DMSO- $\left.d_{6} ; \delta, \mathrm{ppm}\right)$ : 0.98-1.03 (3H, t, $\left.J=7.2 \mathrm{~Hz}, \mathrm{CH}_{2} \mathrm{CH}_{3}\right), 2.33-2.40\left(2 \mathrm{H}, \mathrm{q}, J=7.2 \mathrm{~Hz}, \mathrm{CH}_{2} \mathrm{CH}_{3}\right), 2.47-2.50(4 \mathrm{H}, \mathrm{m}$, piperazine $\left.\mathrm{C}_{3,5}-\mathrm{H}\right), 3.98$ and $4.26\left(\overline{\mathrm{H}}\right.$, two s, piperazine $\left.\mathrm{C}_{2,6}-\mathrm{H}\right), 4.65\left(2 \overline{\mathrm{H}}, \mathrm{s}, \mathrm{COCH}_{2}\right), 7.57-7.62(2 \mathrm{H}, \mathrm{t}$, $J=7.5 \mathrm{~Hz}, \mathrm{Ar}-\mathrm{H}), 7.81-7.87(2 \mathrm{H}, \mathrm{t}, J=7.6 \mathrm{~Hz}, \mathrm{Ar}-\mathrm{H}), 8.14-8.17(2 \mathrm{H}, \mathrm{d}, J=8.7 \overline{\mathrm{Hz}}, \mathrm{Ar}-\mathrm{H}), 8.26-8.29$ $(2 \mathrm{H}, \mathrm{d}, J=8.6 \mathrm{~Hz}, \mathrm{Ar}-\mathrm{H}), 11.19(1 \mathrm{H}, \mathrm{s},-\mathrm{NH}-) ;{ }^{13} \mathrm{C}-\mathrm{NMR}\left(75 \mathrm{MHz}, \mathrm{DMSO}-d_{6} ; \delta, \mathrm{ppm}\right): 12.3\left(\mathrm{CH}_{3}\right), 50.9$ $\left(\mathrm{CH}_{2}\right), 51.1\left(\mathrm{CH}_{2}\right), 51.5\left(\mathrm{CH}_{2}\right), 52.2\left(\mathrm{CH}_{2}\right), 123.2(\mathrm{C}), 125.4(\mathrm{CH}), 126.2(\mathrm{CH}), 129.6(\mathrm{CH}), 130.9(\mathrm{C}), 140.7$ (C), 149.4 (C-9 in 9-aminoacridine), $167.4(\mathrm{C}=\mathrm{O}), 195.0(\mathrm{C}=\mathrm{S})$; HRMS $(\mathrm{m} / \mathrm{z}):[\mathrm{M}+\mathrm{H}]^{+}$calculated for $\mathrm{C}_{22} \mathrm{H}_{24} \mathrm{~N}_{4} \mathrm{OS}_{2}$ : 425.1464, found 425.1459 .

2-(9-Acridinylamino)-2-oxoethyl 4-(4-nitrophenyl)piperazine-1-carbodithioate (4d). Yield: 90.5\%; m.p.: $248.2{ }^{\circ} \mathrm{C}$; FTIR (ATR, $\mathrm{cm}^{-1}$ ): 3238 (amide N-H), 2976 (aliphatic C-H), $1670(\mathrm{C}=\mathrm{O}), 1417-1598(\mathrm{C}=\mathrm{N}$ and $\mathrm{C}=\mathrm{C}$ ), $1213-1280(\mathrm{C}=\mathrm{S}), 752$ (out-of-plane $\mathrm{C}-\mathrm{H}$ bending); ${ }^{1} \mathrm{H}-\mathrm{NMR}\left(300 \mathrm{MHz}, \mathrm{DMSO}-d_{6} ; \delta, \mathrm{ppm}\right.$ ): $3.74\left(4 \mathrm{H}\right.$, s, piperazine $\left.\mathrm{C}_{3,5}-\mathrm{H}\right), 4.19$ and $4.42\left(4 \mathrm{H}\right.$, two bs, piperazine $\left.\mathrm{C}_{2,6}-\mathrm{H}\right), 4.68\left(2 \mathrm{H}, \mathrm{s}, \mathrm{COCH}_{2}\right)$, 6.93-6.96 (2H, d, $J=9.5 \mathrm{~Hz}, \mathrm{Ar}-\mathrm{H}), 7.59-7.63(2 \mathrm{H}, \mathrm{t}, J=6.6 \mathrm{~Hz}, \mathrm{Ar}-\mathrm{H}), 7.82-7.87(2 \mathrm{H}, \mathrm{t}, J=7.7 \mathrm{~Hz}, \mathrm{Ar}-\mathrm{H})$, 8.07-8.10 (2H, d, J = 9.4 Hz, Ar-H), 8.14-8.17 (2H, d, J = 8.7 Hz, Ar-H), 8.27-8.30 (2H, d, J = 8.5 Hz, $\mathrm{Ar}-\mathrm{H}), 11.10(1 \mathrm{H}, \mathrm{s},-\mathrm{NH}-) ;{ }^{13} \mathrm{C}-\mathrm{NMR}\left(75 \mathrm{MHz}, \mathrm{DMSO}-d_{6} ; \delta, \mathrm{ppm}\right): 45.2\left(\mathrm{CH}_{2}\right), 48.9\left(\mathrm{CH}_{2}\right), 50.6\left(\mathrm{CH}_{2}\right)$, $112.3(\mathrm{C}), 123.2(\mathrm{CH}), 125.4(\mathrm{CH}), 126.2(\mathrm{CH}), 126.3(\mathrm{CH}), 129.6(\mathrm{CH}), 130.9(\mathrm{CH}), 137.3(\mathrm{C}), 140.6(\mathrm{C})$, 149.4 (C), 154.2 (C-9 in 9-aminoacridine), $167.3(\mathrm{C}=\mathrm{O}), 195.4(\mathrm{C}=\mathrm{S})$; HRMS $(\mathrm{m} / \mathrm{z})$ : $[\mathrm{M}+\mathrm{H}]^{+}$calculated for $\mathrm{C}_{26} \mathrm{H}_{23} \mathrm{~N}_{5} \mathrm{O}_{3} \mathrm{~S}_{2}: 518.1315$, found 518.1299.

2-(9-Acridinylamino)-2-oxoethyl 4-(2-hydroxyethyl)piperazine-1-carbodithioate (4e). Yield: 84.4\%; m.p.: $219.2{ }^{\circ} \mathrm{C}$; FTIR (ATR, $\mathrm{cm}^{-1}$ ): 3226 (amide N-H), 2978 (aliphatic $\left.\mathrm{C}-\mathrm{H}\right), 1660(\mathrm{C}=\mathrm{O}), 1433-1558(\mathrm{C}=\mathrm{N}$ and $\mathrm{C}=\mathrm{C}$ ), 1228 (C=S), 758 (out-of-plane $\mathrm{C}-\mathrm{H}$ bending); ${ }^{1} \mathrm{H}-\mathrm{NMR}$ (300 MHz, DMSO- $d_{6} ; \delta, \mathrm{ppm}$ ): $2.49-2.50$ $\left(2 \mathrm{H}, \mathrm{t}, \mathrm{J}=1.7 \mathrm{~Hz}, \mathrm{OH}-\mathrm{CH}_{2}-\mathrm{CH}_{2}-\right), 2.61\left(4 \mathrm{H}, \mathrm{s}\right.$, piperazine $\left.\mathrm{C}_{3,5}-\mathrm{H}\right), 3.54\left(2 \mathrm{H}, \mathrm{s}, \mathrm{OH}-\mathrm{CH}_{2}-\mathrm{CH}_{2}-\right), 4.00$ and $4.28\left(4 \mathrm{H}\right.$, s, piperazine $\left.\mathrm{C}_{2,6}-\mathrm{H}\right), 4.64\left(2 \mathrm{H}, \mathrm{s}, \mathrm{COCH}_{2}\right), 7.58-7.62(2 \mathrm{H}, \mathrm{t}, J=7.4 \mathrm{~Hz}, \overline{\mathrm{Ar}-\mathrm{H}}), 7.82-7.87$ $(2 \mathrm{H}, \mathrm{t}, J=7.4 \mathrm{~Hz}, \mathrm{Ar}-\mathrm{H}), 8.14-8.17(2 \mathrm{H}, \mathrm{d}, J=8.6 \overline{\mathrm{Hz}}, \mathrm{Ar}-\mathrm{H}), 8.26-8.28(2 \mathrm{H}, \mathrm{d}, J=8.6 \mathrm{~Hz}, \mathrm{Ar}-\mathrm{H})$, $11.10(1 \mathrm{H}, \mathrm{s},-\mathrm{NH}-) ;{ }^{13} \mathrm{C}-\mathrm{NMR}\left(75 \mathrm{MHz}, \mathrm{DMSO}-d_{6} ; \delta, \mathrm{ppm}\right): 50.2\left(\mathrm{CH}_{2}\right), 51.4\left(\mathrm{CH}_{2}\right), 52.9\left(\mathrm{CH}_{2}\right), 58.8$ $\left(\mathrm{CH}_{2}\right), 59.8\left(\mathrm{CH}_{2}\right), 123.2(\mathrm{C}), 125.4(\mathrm{CH}), 126.2(\mathrm{CH}), 129.6(\mathrm{CH}), 130.9(\mathrm{CH}), 140.6(\mathrm{C}), 149.4(\mathrm{C}-9$ in 9-aminoacridine), $167.4(\mathrm{C}=\mathrm{O}), 195.1(\mathrm{C}=\mathrm{S})$; $\operatorname{HRMS}(\mathrm{m} / \mathrm{z})$ : $[\mathrm{M}+\mathrm{H}]^{+}$calculated for $\mathrm{C}_{22} \mathrm{H}_{24} \mathrm{~N}_{4} \mathrm{O}_{2} \mathrm{~S}_{2}$ : 441.1413, found 441.1406 .

2-(9-Acridinylamino)-2-oxoethyl 4-phenylpiperazine-1-carbodithioate (4f). Yield: 94.5\%; m.p.: oil; FTIR (ATR, $\mathrm{cm}^{-1}$ ): 3352 (amide N-H), 2881 (aliphatic $\left.\mathrm{C}-\mathrm{H}\right), 1660(\mathrm{C}=\mathrm{O}), 1417-1506(\mathrm{C}=\mathrm{N}$ and $\mathrm{C}=\mathrm{C}), 1228$ $(\mathrm{C}=\mathrm{S}), 756-883$ (out-of-plane $\mathrm{C}-\mathrm{H}$ bending); ${ }^{1} \mathrm{H}-\mathrm{NMR}$ (300 MHz, DMSO- $\left.d_{6} ; \delta, \mathrm{ppm}\right): 3.31-3.35(4 \mathrm{H}$, $\mathrm{d}, J=9.4 \mathrm{~Hz}$, piperazine $\left.\mathrm{C}_{3,5}-\mathrm{H}\right), 4.15$ and $4.42\left(4 \mathrm{H}\right.$, two bs, piperazine $\left.\mathrm{C}_{2,6}-\mathrm{H}\right), 4.66\left(2 \mathrm{H}, \mathrm{s}, \mathrm{COCH}_{2}\right)$, 6.78-6.83 $(1 \mathrm{H}, \mathrm{t}, J=7.2 \mathrm{~Hz}, \mathrm{Ar}-\mathrm{H}), 6.94-6.97(2 \mathrm{H}, \mathrm{d}, J=8.0 \mathrm{~Hz}, \mathrm{Ar}-\mathrm{H}), 7.21-7.26(2 \mathrm{H}, \mathrm{t}, J=7 . \overline{\mathrm{Hz}}$, Ar-H), 7.58-7.63 (2H, t, J = 7.6 Hz, Ar-H), 7.82-7.86 (2H, t, $J=6.7 \mathrm{~Hz}, \mathrm{Ar}-\mathrm{H}), 8.14-8.17(2 \mathrm{H}, \mathrm{d}, J=8.7 \mathrm{~Hz}$, $\mathrm{Ar}-\mathrm{H}), 8.27-8.30(2 \mathrm{H}, \mathrm{d}, J=8.5 \mathrm{~Hz}, \mathrm{Ar}-\mathrm{H}), 11.08(1 \mathrm{H}, \mathrm{s},-\mathrm{NH}-) ;{ }^{13} \mathrm{C}-\mathrm{NMR}\left(75 \mathrm{MHz}, \mathrm{DMSO}-d_{6} ; \delta, \mathrm{ppm}\right)$ : $48.1\left(\mathrm{CH}_{2}\right), 50.1\left(\mathrm{CH}_{2}\right), 51.3\left(\mathrm{CH}_{2}\right), 115.9(\mathrm{C}), 119.7(\mathrm{CH}), 123.2(\mathrm{CH}), 125.4(\mathrm{CH}), 126.2(\mathrm{CH}), 129.5(\mathrm{CH})$, 129.6 (CH), $130.9(\mathrm{CH}), 140.6(\mathrm{C}), 149.4(\mathrm{C}), 150.5$ (C-9 in 9-aminoacridine), $167.4(\mathrm{C}=\mathrm{O}), 195.3(\mathrm{C}=\mathrm{S})$; HRMS $(m / z):[\mathrm{M}+\mathrm{H}]^{+}$calculated for $\mathrm{C}_{26} \mathrm{H}_{24} \mathrm{~N}_{4} \mathrm{OS}_{2}: 473.1464$, found 473.1471. 
2-(9-Acridinylamino)-2-oxoethyl 4-methylpiperazine-1-carbodithioate (4g). Yield: 95.3\%; m.p.: $243.2{ }^{\circ} \mathrm{C}$; FTIR (ATR, $\mathrm{cm}^{-1}$ ): 3226 (amide N-H), 2783-2976 (aliphatic $\left.\mathrm{C}-\mathrm{H}\right), 1651(\mathrm{C}=\mathrm{O}), 1435-1571(\mathrm{C}=\mathrm{N}$ and $\mathrm{C}=\mathrm{C}), 1224-1288(\mathrm{C}=\mathrm{S}), 758$ (out-of-plane $\mathrm{C}-\mathrm{H}$ bending); ${ }^{1} \mathrm{H}-\mathrm{NMR}$ (300 MHz, DMSO- $\left.d_{6} ; \delta, \mathrm{ppm}\right): 2.21$ $\left(3 \mathrm{H}, \mathrm{s}, \mathrm{CH}_{3}\right), 2.42-2.45\left(4 \mathrm{H}, \mathrm{t}, J=4.5 \mathrm{~Hz}\right.$, piperazine $\left.\mathrm{C}_{3,5}-\mathrm{H}\right), 3.99$ and $4.27(4 \mathrm{H}$, two s, piperazine $\left.\mathrm{C}_{2,6}-\mathrm{H}\right), 4.61\left(2 \mathrm{H}, \mathrm{s}, \mathrm{COCH}_{2}\right), 7.58-7.63(2 \mathrm{H}, \mathrm{t}, J=7.5 \mathrm{~Hz}, \mathrm{Ar}-\mathrm{H}), 7.82-7.87(2 \mathrm{H}, \mathrm{t}, J=7.3 \mathrm{~Hz}, \mathrm{Ar}-\mathrm{H})$, 8.15-8.18 (2H, d, J = 8.7 Hz, Ar-H), 8.25-8.28 (2H, d, J = 8.6 Hz, Ar-H), $10.94(1 \mathrm{H}, \mathrm{s},-\mathrm{NH}-) ;{ }^{13} \mathrm{C}-\mathrm{NMR}$ (75 MHz, DMSO- $\left.d_{6} ; \delta, \mathrm{ppm}\right): 45.6\left(\mathrm{CH}_{2}\right), 50.3\left(\mathrm{CH}_{3}\right), 51.6\left(\mathrm{CH}_{2}\right), 54.5\left(\mathrm{CH}_{2}\right), 123.2(\mathrm{CH}), 125.3(\mathrm{CH})$, 126.3 (CH), $129.3(\mathrm{CH}), 130.9(\mathrm{CH}), 140.5(\mathrm{CH}), 149.4$ (C-9 in 9-aminoacridine), $167.4(\mathrm{C}=\mathrm{O}), 195.1(\mathrm{C}=\mathrm{S})$; HRMS $(m / z):[\mathrm{M}+\mathrm{H}]^{+}$calculated for $\mathrm{C}_{21} \mathrm{H}_{22} \mathrm{~N}_{4} \mathrm{OS}_{2}$ : 411.1308, found 411.1307.

2-(9-Acridinylamino)-2-oxoethyl 4-(4-methoxyphenyl)piperazine-1-carbodithioate (4h). Yield: 89.4\%; m.p.: $243.8^{\circ} \mathrm{C}$; FTIR (ATR, cm ${ }^{-1}$ ): 3284 (amide N-H), 3057-2945 (aliphatic C-H), 1664 (C=O), 1431-1508 $\left(\mathrm{C}=\mathrm{N}\right.$ and $\mathrm{C}=\mathrm{C}$ ), $1215(\mathrm{C}=\mathrm{S}), 707-756$ (out-of-plane $\mathrm{C}-\mathrm{H}$ bending), ${ }^{1} \mathrm{H}-\mathrm{NMR}$ (300 MHz, DMSO- $d_{6} ; \delta$, ppm): $3.16-3.35\left(4 \mathrm{H}, \mathrm{s}\right.$, piperazine $\left.\mathrm{C}_{3,5}-\mathrm{H}\right), 3.68\left(3 \mathrm{H}, \mathrm{s}, \mathrm{OCH}_{3}\right), 4.14$ and $4.42(4 \mathrm{H}$, two s, piperazine $\left.\mathrm{C}_{2,6}-\mathrm{H}\right), 4.66\left(2 \mathrm{H}, \mathrm{s}, \mathrm{COCH}_{2}\right), 6.81-6.84(2 \mathrm{H}, \mathrm{d}, J=8.5 \mathrm{~Hz}, \overline{\mathrm{Ar}-\mathrm{H}}), 6.90-6.93(2 \mathrm{H}, \mathrm{d}, J=8.4 \mathrm{~Hz}, \mathrm{Ar}-\mathrm{H})$, 7.59-7.64 (2H, t, J = 7.3 Hz, Ar-H), 7.82-7.87 (2H, t, $J=7.4 \mathrm{~Hz}, \mathrm{Ar}-\mathrm{H}), 8.16-8.18(2 \mathrm{H}, \mathrm{d}, J=8.6 \mathrm{~Hz}$, $\mathrm{Ar}-\mathrm{H}), 8.27-8.30(2 \mathrm{H}, \mathrm{d}, J=8.6 \mathrm{~Hz}, \mathrm{Ar}-\mathrm{H}), 10.97(1 \mathrm{H}, \mathrm{s},-\mathrm{NH}-) ;{ }^{13} \mathrm{C}-\mathrm{NMR}\left(75 \mathrm{MHz}, \mathrm{DMSO}-d_{6} ; \delta, \mathrm{ppm}\right)$ : $49.8\left(\mathrm{CH}_{2}\right), 51.4\left(\mathrm{CH}_{2}\right), 55.7\left(\mathrm{CH}_{3}\right), 60.21\left(\mathrm{CH}_{2}\right), 114.8(\mathrm{C}), 118.7(\mathrm{CH}), 123.2(\mathrm{CH}), 125.3(\mathrm{CH}), 126.3$ $(\mathrm{CH}), 129.6(\mathrm{CH}), 130.9(\mathrm{CH}), 140.5(\mathrm{C}), 144.9(\mathrm{C}), 149.4(\mathrm{C}), 153.9$ (C-9 in 9-aminoacridine), $167.4(\mathrm{C}=\mathrm{O})$, $195.3(\mathrm{C}=\mathrm{S})$; HRMS $(\mathrm{m} / z)$ : $[\mathrm{M}+\mathrm{H}]^{+}$calculated for $\mathrm{C}_{27} \mathrm{H}_{26} \mathrm{~N}_{4} \mathrm{O}_{2} \mathrm{~S}_{2}$ : 503.1570, found 503.1547.

2-(9-Acridinylamino)-2-oxoethyl 4-(4-chlorophenyl)piperazine-1-carbodithioate (4i). Yield: 97.0\%; m.p.: $213.6^{\circ} \mathrm{C}$; FTIR (ATR, $\mathrm{cm}^{-1}$ ): 3223 (amide N-H), 2706-2912 (aliphatic C-H), $1672(\mathrm{C}=\mathrm{O}), 1417-1573$ $(\mathrm{C}=\mathrm{N}$ and $\mathrm{C}=\mathrm{C}), 1226(\mathrm{C}=\mathrm{S}), 815$ (para aromatic substitution), 756 (out-of-plane $\mathrm{C}-\mathrm{H}$ bending); ${ }^{1} \mathrm{H}-\mathrm{NMR}$ (300 MHz, DMSO- $\left.d_{6} ; \delta, \mathrm{ppm}\right): 2.44-2.50\left(4 \mathrm{H}, \mathrm{q}, J=5.8 \mathrm{~Hz}\right.$, piperazine $\left.\mathrm{C}_{3,5}-\mathrm{H}\right), 4.02$ and $4.45(4 \mathrm{H}$, two bs, piperazine $\left.\mathrm{C}_{2,6}-\mathrm{H}\right), 4.60\left(2 \mathrm{H}, \mathrm{s}, \mathrm{COCH}_{2}\right), 6.97-7.00(2 \mathrm{H}, \mathrm{d}, J=9.1 \mathrm{~Hz}, \mathrm{Ar}-\mathrm{H}), 7.25-7.28(2 \mathrm{H}$, $\mathrm{d}, J=9.0 \mathrm{~Hz}, \mathrm{Ar}-\mathrm{H}), 7.57-7.62(2 \mathrm{H}, \mathrm{t}, J=7.5 \overline{\mathrm{Hz}}, \mathrm{Ar}-\mathrm{H}), 7.81-7.85(2 \mathrm{H}, \mathrm{t}, J=5.9 \mathrm{~Hz}, \mathrm{Ar}-\mathrm{H}), 8.14-8.17$ $(2 \mathrm{H}, \mathrm{d}, J=8.7 \mathrm{~Hz}, \mathrm{Ar}-\mathrm{H}), 8.27-8.30(2 \mathrm{H}, \mathrm{d}, J=8.6 \mathrm{~Hz}, \mathrm{Ar}-\mathrm{H}), 11.22(1 \mathrm{H}, \mathrm{s},-\mathrm{NH}-) ;{ }^{13} \mathrm{C}-\mathrm{NMR}(75 \mathrm{MHz}$, DMSO- $\left.d_{6} ; \delta, \mathrm{ppm}\right): 45.6\left(\mathrm{CH}_{2}\right), 49.8\left(\mathrm{CH}_{2}\right), 51.0\left(\mathrm{CH}_{2}\right), 117.3(\mathrm{CH}), 118.0(\mathrm{CH}), 123.2(\mathrm{CH}), 125.2(\mathrm{CH})$, 126.2 (CH), $129.2(\mathrm{CH}), 129.3(\mathrm{CH}), 129.6(\mathrm{CH}), 130.9(\mathrm{CH}), 140.7(\mathrm{C}), 149.4$ (C-9 in 9-aminoacridine), 167.4 $(\mathrm{C}=\mathrm{O}), 195.3(\mathrm{C}=\mathrm{S})$; HRMS $(\mathrm{m} / \mathrm{z})$ : $[\mathrm{M}+\mathrm{H}]^{+}$calculated for $\mathrm{C}_{26} \mathrm{H}_{23} \mathrm{ClN}_{4} \mathrm{OS}_{2}$ : 507.1075, found 507.1069 .

2-(9-Acridinylamino)-2-oxoethyl 4-(pyrimidin-2-yl)piperazine-1-carbodithioate (4j). Yield: 84.3\%; m.p.: $248.4^{\circ} \mathrm{C}$; FTIR (ATR, $\mathrm{cm}^{-1}$ ): 3263 (amide $\left.\mathrm{N}-\mathrm{H}\right), 2852-2897$ (aliphatic $\left.\mathrm{C}-\mathrm{H}\right), 1662(\mathrm{C}=\mathrm{O}), 1425-1583$ $(\mathrm{C}=\mathrm{N}$ and $\mathrm{C}=\mathrm{C}), 1222-1257(\mathrm{C}=\mathrm{S}), 759-792$ (out-of-plane $\mathrm{C}-\mathrm{H}$ bending); ${ }^{1} \mathrm{H}-\mathrm{NMR}$ (300 MHz, DMSO- $d_{6}$; $\delta, \mathrm{ppm}): 3.91\left(4 \mathrm{H}, \mathrm{s}\right.$, piperazine $\left.\mathrm{C}_{3,5}-\mathrm{H}\right), 4.13$ and $4.38\left(4 \mathrm{H}\right.$, two s, piperazine $\left.\mathrm{C}_{2,6}-\mathrm{H}\right), 4.68(2 \mathrm{H}, \mathrm{s}$, $\left.\mathrm{COCH}_{2}\right), 6.67-6.70(1 \mathrm{H}, \mathrm{t}, J=4.7 \mathrm{~Hz}, \mathrm{Ar}-\mathrm{H}), 7.58-7.63(2 \mathrm{H}, \mathrm{t}, J=7.6 \mathrm{~Hz}, \mathrm{Ar}-\mathrm{H}), 7.82-7.87(2 \mathrm{H}, \mathrm{t}$, $J=7.4 \mathrm{~Hz}, \mathrm{Ar}-\mathrm{H}), 8.14-8.18(2 \mathrm{H}, \mathrm{d}, J=8.7 \mathrm{~Hz}, \mathrm{Ar}-\mathrm{H}), 8.27-8.30(2 \mathrm{H}, \mathrm{d}, J=8.6 \mathrm{~Hz}, \mathrm{Ar}-\mathrm{H}), 8.39-8.41$ $(2 \mathrm{H}, \mathrm{d}, J=4.7 \mathrm{~Hz}, \mathrm{Ar}-\mathrm{H}), 11.16(1 \mathrm{H}, \mathrm{s},-\mathrm{NH}-) ;{ }^{13} \mathrm{C}-\mathrm{NMR}\left(75 \mathrm{MHz}, \mathrm{DMSO}-d_{6} ; \delta, \mathrm{ppm}\right): 43.0\left(\mathrm{CH}_{2}\right), 49.8$ $\left(\mathrm{CH}_{2}\right), 51.2\left(\mathrm{CH}_{2}\right), 111.1(\mathrm{C}), 123.2(\mathrm{CH}), 125.4(\mathrm{CH}), 126.2(\mathrm{CH}), 129.6(\mathrm{CH}), 130.9(\mathrm{CH}), 140.6(\mathrm{C}), 149.4$ $(\mathrm{CH}), 158.48(\mathrm{C}), 161.3\left(\mathrm{C}-9\right.$ in 9-aminoacridine), $167.4(\mathrm{C}=\mathrm{O}), 195.4(\mathrm{C}=\mathrm{S})$. HRMS $(m / z):[\mathrm{M}+\mathrm{H}]^{+}$ calculated for $\mathrm{C}_{24} \mathrm{H}_{22} \mathrm{~N}_{6} \mathrm{OS}_{2}: 475.1369$, found 475.1366 .

2-(9-Acridinylamino)-2-oxoethyl 4-benzylpiperazine-1-carbodithioate (4k). Yield: $91.4 \%$; m.p.: $222.3^{\circ} \mathrm{C}$; FTIR (ATR, $\mathrm{cm}^{-1}$ ): 3248 (amide $\left.\mathrm{N}-\mathrm{H}\right), 2818-2908$ (aliphatic $\left.\mathrm{C}-\mathrm{H}\right), 1668(\mathrm{C}=\mathrm{O}), 1417-1510(\mathrm{C}=\mathrm{N}$ and $\mathrm{C}=\mathrm{C})$, $1230(\mathrm{C}=\mathrm{S}), 731-756$ (out-of-plane $\mathrm{C}-\mathrm{H}$ bending); ${ }^{1} \mathrm{H}-\mathrm{NMR}\left(300 \mathrm{MHz}, \mathrm{DMSO}-d_{6} ; \delta, \mathrm{ppm}\right): 3.53$ and $3.99\left(4 \mathrm{H}\right.$, two bs, piperazine $\left.\mathrm{C}_{2}, 6-\mathrm{H}\right), 4.28\left(2 \mathrm{H}, \mathrm{s}, \mathrm{C}_{6} \mathrm{H}_{5}-\mathrm{CH}_{2}-\right), 4.62\left(2 \mathrm{H}, \mathrm{s}, \mathrm{COCH}_{2}\right), 7.26-7.32(5 \mathrm{H}, \mathrm{m}$, Ar-H), 7.57-7.62 (2H, t, J = 7.5 Hz, Ar-H), 7.82-7.87 (2H, t, J = 7.5 Hz, Ar-H), 8.14-8.17 (2H, d, J = 8.7 Hz, Ar-H), 8.25-8.28 (2H, d, J = 8.6 Hz, Ar-H), $11.05(1 \mathrm{H}, \mathrm{s},-\mathrm{NH}-) ;{ }^{13} \mathrm{C}-\mathrm{NMR}\left(75 \mathrm{MHz}, \mathrm{DMSO}-d_{6} ; \delta, \mathrm{ppm}\right)$ : 50.4 $\left(\mathrm{CH}_{2}\right), 51.7\left(\mathrm{CH}_{2}\right), 52.4\left(\mathrm{CH}_{2}\right), 61.8\left(\mathrm{CH}_{2}\right), 123.2(\mathrm{C}), 125.3(\mathrm{CH}), 126.2(\mathrm{CH}), 127.6(\mathrm{CH}), 128.7(\mathrm{CH})$, 
$129.4(\mathrm{CH}), 129.6(\mathrm{CH}), 130.9(\mathrm{CH}), 138.0(\mathrm{C}), 140.6(\mathrm{C}), 149.4$ (C-9 in 9-aminoacridine), $167.4(\mathrm{C}=\mathrm{O})$, 195.0 (C=S); HRMS ( $m / z)$ : $[\mathrm{M}+\mathrm{H}]^{+}$calculated for $\mathrm{C}_{27} \mathrm{H}_{26} \mathrm{~N}_{4} \mathrm{OS}_{2}$ : 487.1621, found 487.1622.

2-(9-Acridin-ylamino)-2-oxoethyl 4-cyclohexylpiperazine-1-carbodithioate (41). Yield: 88.4\%; m.p.: 225.4 ${ }^{\circ} \mathrm{C}$; FTIR (ATR, $\mathrm{cm}^{-1}$ ): 3309 (amide N-H), 2357-2931 (aliphatic C-H), 1676 (C=O), 1469-1566 (C=N and $\mathrm{C}=\mathrm{C}), 1230-1274(\mathrm{C}=\mathrm{S}), 754-866$ (out-of-plane $\mathrm{C}-\mathrm{H}$ bending); ${ }^{1} \mathrm{H}-\mathrm{NMR}\left(300 \mathrm{MHz}, \mathrm{DMSO}-d_{6} ; \delta, \mathrm{ppm}\right.$ ): 1.03-1.18 (6H, m, cyclohexyl $\left.-\mathrm{CH}_{2}-\right), 1.54-1.57(1 \mathrm{H}, \mathrm{d}, J=11.2 \mathrm{~Hz}$, cyclohexyl $-\mathrm{CH}-), 1.72(4 \mathrm{H}, \mathrm{s}$, cyclohexyl $\left.-\mathrm{CH}_{2}-\right), 2.60\left(4 \mathrm{H}\right.$, s, piperazine $\left.\mathrm{C}_{3,5}-\mathrm{H}\right), 3.94$ and $4.24\left(4 \mathrm{H}\right.$, two bs, piperazine $\left.\mathrm{C}_{2,6}-\mathrm{H}\right), 4.62$ $\left(2 \mathrm{H}, \mathrm{s}, \mathrm{COCH}_{2}\right), 7.57-7.62(2 \mathrm{H}, \mathrm{t}, J=7.5 \mathrm{~Hz}, \mathrm{Ar}-\mathrm{H}), 7.82-7.87(2 \mathrm{H}, \mathrm{t}, J=7.6 \mathrm{~Hz}, \mathrm{Ar}-\mathrm{H}), 8.14-8.17(2 \mathrm{H}$, $\mathrm{d}, J=8.7 \mathrm{~Hz}, \mathrm{Ar}-\mathrm{H}), 8.25-8.28(2 \mathrm{H}, \mathrm{d}, J=8.6 \mathrm{~Hz}, \mathrm{Ar}-\mathrm{H}), 11.04(1 \mathrm{H}, \mathrm{s},-\mathrm{NH}-) ;{ }^{13} \mathrm{C}-\mathrm{NMR}(75 \mathrm{MHz}$, DMSO- $\left.d_{6} ; \delta, \mathrm{ppm}\right): 25.7\left(\mathrm{CH}_{2}\right), 26.3\left(\mathrm{CH}_{2}\right), 28.8\left(\mathrm{CH}_{2}\right), 48.6\left(\mathrm{CH}_{2}\right), 50.9\left(\mathrm{CH}_{2}\right), 52.2\left(\mathrm{CH}_{2}\right), 62.7(\mathrm{CH})$, 123.2 (C), $125.4(\mathrm{CH}), 126.2(\mathrm{CH}), 129.6(\mathrm{CH}), 130.9(\mathrm{CH}), 140.6(\mathrm{C}), 149.4$ (C-9 in 9-aminoacridine), 167.4 $(\mathrm{C}=\mathrm{O}), 194.7(\mathrm{C}=\mathrm{S})$; HRMS $(\mathrm{m} / \mathrm{z}):[\mathrm{M}+\mathrm{H}]^{+}$calculated for $\mathrm{C}_{26} \mathrm{H}_{30} \mathrm{~N}_{4} \mathrm{OS}_{2}$ : 479.1934, found 479.1929.

2-(9-Acridinylamino)-2-oxoethyl 4-benzhydrylpiperazine-1-carbodithioate (4m). Yield: $84.3 \%$; m.p.: $137.0^{\circ} \mathrm{C}$; FTIR (ATR, $\mathrm{cm}^{-1}$ ): 3246 (amide N-H), 2355-2920 (aliphatic C-H), 1656 (C=O), 1427-1571 (C=N and $\mathrm{C}=\mathrm{C}), 1226-1284(\mathrm{C}=\mathrm{S}), 705-758$ (out-of-plane $\mathrm{C}-\mathrm{H}$ bending); ${ }^{1} \mathrm{H}-\mathrm{NMR}\left(300 \mathrm{MHz}, \mathrm{DMSO}-d_{6} ; \delta, \mathrm{ppm}\right.$ ): 2.44-2.50 $\left(4 \mathrm{H}, \mathrm{d}, J=5.8 \mathrm{~Hz}\right.$, piperazine $\left.\mathrm{C}_{3,5}-\mathrm{H}\right), 4.02$ and $4.28\left(4 \mathrm{H}\right.$, two s, piperazine $\left.\mathrm{C}_{2,6}-\mathrm{H}\right), 4.40(1 \mathrm{H}$, $\left.\mathrm{s}, \mathrm{C}_{12} \mathrm{H}_{10}-\underline{\mathrm{CH}}-\right), 4.60\left(2 \mathrm{H}, \mathrm{s}, \mathrm{COCH}_{2}\right), 7.18-7.23(3 \mathrm{H}, \mathrm{t}, J=7.0 \mathrm{~Hz}, \mathrm{Ar}-\mathrm{H}), 7.29-7.32(4 \mathrm{H}, \mathrm{t}, J=7.4 \mathrm{~Hz}$, $\mathrm{Ar}-\mathrm{H}), 7.44(4 \mathrm{H}, \mathrm{s}, \mathrm{Ar}-\mathrm{H}), 7.57-7.62(2 \mathrm{H}, \mathrm{t}, J=7.6 \mathrm{~Hz}, \mathrm{Ar}-\mathrm{H}), 7.82-7.88(2 \mathrm{H}, \mathrm{m}, \mathrm{Ar}-\mathrm{H}), 7.15-7.18(2 \mathrm{H}, \mathrm{d}$, $J=8.7 \mathrm{~Hz}, \mathrm{Ar}-\mathrm{H}), 7.24-7.27(2 \mathrm{H}, \mathrm{d}, J=8.6 \mathrm{~Hz}, \mathrm{Ar}-\mathrm{H}), 11.02(1 \mathrm{H}, \mathrm{s},-\mathrm{NH}-) ;{ }^{13} \mathrm{C}-\mathrm{NMR}\left(75 \mathrm{MHz}, \mathrm{DMSO}-d_{6}\right.$; 8, ppm): $51.4\left(\mathrm{CH}_{2}\right), 60.7\left(\mathrm{CH}_{2}\right), 72.7\left(\mathrm{CH}_{2}\right), 74.6(\mathrm{CH}), 117.9(\mathrm{C}), 123.1(\mathrm{CH}), 125.4(\mathrm{CH}), 126.3(\mathrm{CH})$, 127.6 (CH), $128.1(\mathrm{CH}), 129.1(\mathrm{CH}), 131.2(\mathrm{CH}), 140.9(\mathrm{C}), 142.6(\mathrm{C}), 149.1$ (C-9 in 9-aminoacridine), 167.4 $(\mathrm{C}=\mathrm{O}), 195.1(\mathrm{C}=\mathrm{S})$; HRMS $(\mathrm{m} / \mathrm{z})$ : $[\mathrm{M}+\mathrm{H}]^{+}$calculated for $\mathrm{C}_{33} \mathrm{H}_{30} \mathrm{~N}_{4} \mathrm{OS}_{2}$ : 563.1934, found 563.1924.

2-(9-Acridinylamino)-2-oxoethyl 4-(4-(trifluoromethyl)benzyl)piperazine-1-carbodithioate (4n). Yield: 87.7\%; m.p.: $161.5^{\circ} \mathrm{C}$; FTIR (ATR, $\mathrm{cm}^{-1}$ ): 3232 (amide N-H), 2814-2920 (aliphatic C-H), $1653(\mathrm{C}=\mathrm{O}), 1415-1510$ $(\mathrm{C}=\mathrm{N}$ and $\mathrm{C}=\mathrm{C}), 1219(\mathrm{C}=\mathrm{S}), 756$ (out-of-plane $\mathrm{C}-\mathrm{H}$ bending); ${ }^{1} \mathrm{H}-\mathrm{NMR}\left(300 \mathrm{MHz}, \mathrm{DMSO}-d_{6} ; \delta, \mathrm{ppm}\right)$ : 2.48-2.52 $\left(4 \mathrm{H}, \mathrm{t}, J=5.10 \mathrm{~Hz}\right.$, piperazine $\left.\mathrm{C}_{3,5}-\mathrm{H}\right), 3.62$ and $4.00\left(4 \mathrm{H}\right.$, two s, piperazine $\left.\mathrm{C}_{2,6}-\mathrm{H}\right), 4.29(2 \mathrm{H}$, s, $\left.\mathrm{C}_{6} \mathrm{H}_{5}-\mathrm{CH}_{2}-\right), 4.62\left(2 \mathrm{H}, \mathrm{s}, \mathrm{COCH}_{2}\right), 7.55-7.57(2 \mathrm{H}, \mathrm{d}, J=7.9 \mathrm{~Hz}, \mathrm{Ar}-\mathrm{H}), 7.60-7.62(2 \mathrm{H}, \mathrm{d}, J=7.7 \mathrm{~Hz}$, Ar-H), 7.68-7.71 (2H, d, $J=8.2 \mathrm{~Hz} \mathrm{Ar}-\mathrm{H}), 7.82-7.87(2 \mathrm{H}, \mathrm{t}, J=7.8 \mathrm{~Hz}, \mathrm{Ar}-\mathrm{H}), 7.14-7.17(2 \mathrm{H}, \mathrm{d}, J=8.8 \mathrm{~Hz}$, $\mathrm{Ar}-\mathrm{H}), 7.25-7.27(2 \mathrm{H}, \mathrm{d}, J=8.6 \mathrm{~Hz}, \mathrm{Ar}-\mathrm{H}), 11.01(1 \mathrm{H}, \mathrm{s},-\mathrm{NH}-) ;{ }^{13} \mathrm{C}-\mathrm{NMR}\left(75 \mathrm{MHz}\right.$, DMSO- $\left.d_{6} ; \delta, \mathrm{ppm}\right)$ : $52.4\left(\mathrm{CH}_{2}\right), 60.8\left(\mathrm{CH}_{2}\right), 72.7\left(\mathrm{CH}_{2}\right), 125.0\left(\mathrm{q}, J=214.9 \mathrm{~Hz}, \mathrm{CF}_{3}\right), 123.2(\mathrm{CH}), 125.3(\mathrm{CH}), 125.6(\mathrm{q}, J=3.6 \mathrm{~Hz}$, trifluoromethylphenyl $\left.\mathrm{C}_{3,3^{\prime}}\right), 126.2(\mathrm{CH}), 129.6(\mathrm{CH}), 129.9\left(\mathrm{q}, J=39.8 \mathrm{~Hz}\right.$, trifluoromethylphenyl $\left.\mathrm{C}_{4}\right)$, 130.0 (CH), $130.1(\mathrm{C}), 140.6(\mathrm{C}), 143.2(\mathrm{C}), 149.4$ (C-9 in 9-aminoacridine), $167.4(\mathrm{C}=\mathrm{O}), 195.1(\mathrm{C}=\mathrm{S})$; HRMS $(m / z):[\mathrm{M}+\mathrm{H}]^{+}$calculated for $\mathrm{C}_{28} \mathrm{H}_{25} \mathrm{~F}_{3} \mathrm{~N}_{4} \mathrm{OS}_{2}$ : 555.1495, found 555.1493.

2-(9-Acridinylamino)-2-oxoethyl 4-(4-methylbenzyl)piperazine-1-carbodithioate (4o). Yield: 91.0\%; m.p.: 205.8 ${ }^{\circ} \mathrm{C}$; FTIR (ATR, $\mathrm{cm}^{-1}$ ): 3255 (amide N-H), 2792-2902 (aliphatic C-H), 1654 (C=O), 1435-1508 $(\mathrm{C}=\mathrm{N}$ and $\mathrm{C}=\mathrm{C}), 1220(\mathrm{C}=\mathrm{S}), 759$ (out-of-plane $\mathrm{C}-\mathrm{H}$ bending); ${ }^{1} \mathrm{H}-\mathrm{NMR}$ (300 MHz, DMSO- $d_{6} ; \delta, \mathrm{ppm}$ ): $2.27\left(3 \mathrm{H}, \mathrm{s},-\mathrm{CH}_{3}\right), 2.47-2.50\left(4 \mathrm{H}, \mathrm{m}\right.$, piperazine $\left.\mathrm{C}_{3,5}-\mathrm{H}\right), 3.47\left(2 \mathrm{H}, \mathrm{s}, \mathrm{C}_{6} \mathrm{H}_{5}-\mathrm{CH}_{2}\right), 3.98$ and $4.26(4 \mathrm{H}$, two s, piperazine $\left.\mathrm{C}_{2,6}-\mathrm{H}\right), 4.63\left(2 \mathrm{H}, \mathrm{s}, \mathrm{COCH}_{2}\right), 7.11-7.14(2 \mathrm{H}, \mathrm{d}, J=7.9 \mathrm{~Hz}, \mathrm{Ar}-\mathrm{H}), 7.18-7.20(2 \mathrm{H}$, $\mathrm{d}, J=7.9 \mathrm{~Hz}, \mathrm{Ar}-\mathrm{H}), 7.56-7.61(2 \mathrm{H}, \mathrm{t}, J=7.5 \mathrm{~Hz}, \mathrm{Ar}-\mathrm{H}), 7.86-7.81(2 \mathrm{H}, \mathrm{t}, J=7.4 \mathrm{~Hz}, \mathrm{Ar}-\mathrm{H}), 8.14-8.17$ $(2 \mathrm{H}, \mathrm{d}, J=8.7 \mathrm{~Hz}, \mathrm{Ar}-\mathrm{H}), 8.25-8.28(2 \mathrm{H}, \mathrm{d}, J=8.6 \mathrm{~Hz}, \mathrm{Ar}-\mathrm{H}), 11.15(1 \mathrm{H}, \mathrm{s},-\mathrm{NH}-) ;{ }^{13} \mathrm{C}-\mathrm{NMR}(75 \mathrm{MHz}$, DMSO- $\left.d_{6} ; \delta, \mathrm{ppm}\right): 21.2\left(\mathrm{CH}_{3}\right), 50.4\left(\mathrm{CH}_{2}\right), 51.6\left(\mathrm{CH}_{2}\right), 52.4\left(\mathrm{CH}_{2}\right), 61.5\left(\mathrm{CH}_{2}\right), 123.2(\mathrm{C}), 125.4(\mathrm{CH})$, $126.2(\mathrm{CH}), 129.3(\mathrm{CH}), 129.4(\mathrm{CH}), 129.6(\mathrm{CH}), 130.9(\mathrm{CH}), 134.8(\mathrm{C}), 36.7(\mathrm{C}), 140.6(\mathrm{C}), 149.4(\mathrm{C}-9$ in 9-aminoacridine), $167.4(\mathrm{C}=\mathrm{O}), 195.0(\mathrm{C}=\mathrm{S})$; $\operatorname{HRMS}(\mathrm{m} / z)$ : $[\mathrm{M}+\mathrm{H}]^{+}$calculated for $\mathrm{C}_{28} \mathrm{H}_{28} \mathrm{~N}_{4} \mathrm{OS}_{2}$ : 501.1777, found 501.1774 .

2-(9-Acridinylamino)-2-oxoethyl 4-(4-fluorophenyl)piperazine-1-carbodithioate (4p). Yield: 96.4\%; m.p.: $227.7^{\circ} \mathrm{C}$; FTIR (ATR, cm ${ }^{-1}$ ): 3257 (amide N-H), 2800-2906 (aliphatic C-H), $1653(\mathrm{C}=\mathrm{O}), 1433(\mathrm{C}=\mathrm{N}$ and $\mathrm{C}=\mathrm{C}), 1213(\mathrm{C}=\mathrm{S}), 1029(\mathrm{C}-\mathrm{F}), 825$ (1,4-disubstituted benzene), 756 (out-of-plane $\mathrm{C}-\mathrm{H}$ bending); 
${ }^{1} \mathrm{H}-\mathrm{NMR}\left(300 \mathrm{MHz}, \mathrm{DMSO}-d_{6} ; \delta, \mathrm{ppm}\right): 3.24-3.27\left(4 \mathrm{H}, \mathrm{t}, J=4.8 \mathrm{~Hz}\right.$, piperazine $\left.\mathrm{C}_{3,5}-\mathrm{H}\right), 4.16$ and $4.39\left(4 \mathrm{H}\right.$, two s, piperazine $\left.\mathrm{C}_{2,6}-\mathrm{H}\right), 4.67\left(2 \mathrm{H}, \mathrm{s}, \mathrm{COCH}_{2}\right), 6.94-6.99(2 \mathrm{H}, \mathrm{m}, \mathrm{Ar}-\mathrm{H}), 7.04-7.10(2 \mathrm{H}, \mathrm{t}$, $J=8.9 \mathrm{~Hz}, \mathrm{Ar}-\mathrm{H}), 7.58-7.63(2 \mathrm{H}, \mathrm{t}, J=7.6 \mathrm{~Hz}, \mathrm{Ar}-\mathrm{H}), 7.82-7.87(2 \mathrm{H}, \mathrm{t}, J=7.7 \mathrm{~Hz}, \mathrm{Ar}-\mathrm{H}), 8.15-8.18$ $(2 \mathrm{H}, \mathrm{d}, J=8.7 \mathrm{~Hz}, \mathrm{Ar}-\mathrm{H}), 8.27-8.30(2 \mathrm{H}, \mathrm{d}, J=8.6 \mathrm{~Hz}, \mathrm{Ar}-\mathrm{H}), 11.07(1 \mathrm{H}, \mathrm{s},-\mathrm{NH}-) ;{ }^{13} \mathrm{C}-\mathrm{NMR}(75 \mathrm{MHz}$, DMSO- $\left.d_{6} ; \delta, \mathrm{ppm}\right): 49.0\left(\mathrm{CH}_{2}\right), 50.1\left(\mathrm{CH}_{2}\right), 51.36\left(\mathrm{CH}_{2}\right), 115.9\left(\mathrm{~d}, J=21.8 \mathrm{~Hz}\right.$, fluorophenyl $\left.\mathrm{C}_{3,3^{\prime}}\right), 117.9$ $\left(\mathrm{d}, J=7.5 \mathrm{~Hz}\right.$, fluorophenyl $\left.\mathrm{C}_{2,2^{\prime}}\right), 123.2(\mathrm{CH}), 125.4(\mathrm{CH}), 126.2(\mathrm{CH}), 129.6(\mathrm{CH}), 130.9(\mathrm{CH}), 140.6$ $(\mathrm{CH}), 147.4\left(\mathrm{~d}, J=2.3 \mathrm{~Hz}\right.$, fluorophenyl $\left.\mathrm{C}_{1}\right), 149.4$ (C-9 in 9-aminoacridine), $156.8(\mathrm{~d}, J=234.8 \mathrm{~Hz}$, fluorophenyl $\left.\mathrm{C}_{4}\right), 167.4(\mathrm{C}=\mathrm{O}), 195.3(\mathrm{C}=\mathrm{S})$; $\operatorname{HRMS}(\mathrm{m} / z)$ : $[\mathrm{M}+\mathrm{H}]^{+}$calculated for $\mathrm{C}_{26} \mathrm{H}_{23} \mathrm{FN}_{4} \mathrm{OS}_{2}$ : 490.62, found 491.13; HRMS ( $m / z)$ : $[\mathrm{M}+\mathrm{H}]^{+}$calculated for $\mathrm{C}_{26} \mathrm{H}_{23} \mathrm{FN}_{4} \mathrm{OS}_{2}$ : 491.1370, found 491.1354.

2-(9-Acridinylamino)-2-oxoethyl 4-(furan-2-carbonyl)piperazine-1-carbodithioate (4q). Yield: 95.5\%; m.p.: $218.9^{\circ} \mathrm{C}$; FTIR (ATR, $\mathrm{cm}^{-1}$ ): 3255 (amide N-H), 2895-2995 (aliphatic C-H), 1653 (furan-2-carbonyl), 1616 (amide $\mathrm{C}=\mathrm{O}), 1423\left(\mathrm{C}=\mathrm{N}\right.$ and $\mathrm{C}=\mathrm{C}$ ), $1215(\mathrm{C}=\mathrm{S}), 758$ (out-of-plane $\mathrm{C}-\mathrm{H}$ bending); ${ }^{1} \mathrm{H}-\mathrm{NMR}$ (300 MHz, DMSO- $\left.d_{6} ; \delta, \mathrm{ppm}\right): 3.88\left(4 \mathrm{H}\right.$, s, piperazine $\left.\mathrm{C}_{3,5}-\mathrm{H}\right), 4.12$ and $4.36(4 \mathrm{H}$, two s, piperazine $\left.-\mathrm{CH}_{2}\right), 4.68\left(2 \mathrm{H}, \mathrm{s}, \mathrm{COCH}_{2}\right), 6.64-6.65(1 \mathrm{H}, \mathrm{m}, \mathrm{Ar}-\mathrm{H}), 7.07-7.08(2 \mathrm{H}, \mathrm{d}, J=3.4 \mathrm{~Hz}, \mathrm{Ar}-\mathrm{H}), 7.57-7.63(2 \mathrm{H}$, $\mathrm{t}, J=7.6 \mathrm{~Hz}, \mathrm{Ar}-\mathrm{H}), 7.81-7.87(3 \mathrm{H}, \mathrm{t}, J=8.1 \mathrm{~Hz}, \mathrm{Ar}-\mathrm{H}), 8.14-8.17(2 \mathrm{H}, \mathrm{d}, J=8.7 \mathrm{~Hz}, \mathrm{Ar}-\mathrm{H}), 8.27-8.30$ $(2 \mathrm{H}, \mathrm{d}, J=8.6 \mathrm{~Hz}, \mathrm{Ar}-\mathrm{H}), 11.21(1 \mathrm{H}, \mathrm{s},-\mathrm{NH}-) ;{ }^{13} \mathrm{C}-\mathrm{NMR}\left(75 \mathrm{MHz}, \mathrm{DMSO}-d_{6} ; \delta, \mathrm{ppm}\right): 49.6\left(\mathrm{CH}_{2}\right), 51.1$ $\left(\mathrm{CH}_{2}\right), 111.9(\mathrm{C}), 116.6(\mathrm{CH}), 123.2(\mathrm{CH}), 125.4(\mathrm{CH}), 126.2(\mathrm{CH}), 129.6(\mathrm{CH}), 130.9(\mathrm{CH}), 140.6(\mathrm{CH})$, 145.6 (C), 147.1 (C), 149.4 (C), 159.0 (C-9 in 9-aminoacridine), 167.4 (C=O), 195.7 (C=S); HRMS ( $\mathrm{m} / \mathrm{z}$ ): $[\mathrm{M}+\mathrm{H}]^{+}$calculated for $\mathrm{C}_{25} \mathrm{H}_{22} \mathrm{~N}_{4} \mathrm{O}_{3} \mathrm{~S}_{2}$ : 491.1206, found 491.1186.

2-(9-Acridinylamino)-2-oxoethyl morpholine-4-carbodithioate (4r). Yield: $89.2 \%$; m.p.: $237.8{ }^{\circ} \mathrm{C}$; FTIR (ATR, $\mathrm{cm}^{-1}$ ): 3232 (amide N-H), 2864-2949 (aliphatic $\mathrm{C}-\mathrm{H}$ ), 1653 (amide $\mathrm{C}=\mathrm{O}$ ), 1417-1423 (C=N and $\mathrm{C}=\mathrm{C}$ ), $1273(\mathrm{C}=\mathrm{S}), 1001$ ( $\mathrm{C}-\mathrm{O}-\mathrm{C}$ of morpholine), 864-758 (out-of-plane $\mathrm{C}-\mathrm{H}$ bending); ${ }^{1} \mathrm{H}-\mathrm{NMR}$ (300 MHz, DMSO- $\left.d_{6} ; \delta, \mathrm{ppm}\right): 3.71-3.72\left(4 \mathrm{H}, \mathrm{d}, J=4.3 \mathrm{~Hz}\right.$, morpholine $\left.\mathrm{C}_{3.5}-\mathrm{H}\right), 4.01$ and $4.25(4 \mathrm{H}$, two bs, morpholine $\left.\mathrm{C}_{2,6}-\mathrm{H}\right), 4.65\left(2 \mathrm{H}, \mathrm{s}, \mathrm{COCH}_{2}\right), 7.58-7.63(2 \mathrm{H}, \mathrm{t}, J=7.5 \mathrm{~Hz}, \mathrm{Ar}-\mathrm{H}), 7.82-7.87(2 \mathrm{H}, \mathrm{t}$, $J=7.6 \mathrm{~Hz}, \mathrm{Ar}-\mathrm{H}), 8.15-8.18(2 \mathrm{H}, \mathrm{d}, J=8.7 \mathrm{\textrm {Hz }}, \mathrm{Ar}-\mathrm{H}), 8.26-8.29(2 \mathrm{H}, \mathrm{d}, J=8.6 \mathrm{~Hz}, \mathrm{Ar}-\mathrm{H}), 11.06(1 \mathrm{H}$, $\mathrm{s},-\mathrm{NH}-) ;{ }^{13} \mathrm{C}-\mathrm{NMR}\left(75 \mathrm{MHz}, \mathrm{DMSO}-d_{6} ; \delta, \mathrm{ppm}\right): 50.9\left(\mathrm{CH}_{2}\right), 51.8\left(\mathrm{CH}_{2}\right), 66.1\left(\mathrm{CH}_{2}\right), 123.2(\mathrm{C}), 125.3$ $(\mathrm{CH}), 126.2(\mathrm{CH}), 129.6(\mathrm{CH}), 130.9(\mathrm{CH}), 140.6(\mathrm{C}), 149.4$ (C-9 in 9-aminoacridine), $167.4(\mathrm{C}=\mathrm{O}), 195.7$ $(\mathrm{C}=\mathrm{S})$; HRMS $(m / z)$ : $[\mathrm{M}+\mathrm{H}]^{+}$calculated for $\mathrm{C}_{20} \mathrm{H}_{19} \mathrm{~N}_{3} \mathrm{O}_{2} \mathrm{~S}_{2}: 398.0991$, found 398.0977.

2-(9-Acridinylamino)-2-oxoethyl piperidine-1-carbodithioate (4s). Yield: 84.4\%; m.p.: $265.4{ }^{\circ} \mathrm{C}$; FTIR (ATR, $\mathrm{cm}^{-1}$ ): 3255 (amide $\mathrm{N}-\mathrm{H}$ ), 2904-2983 (aliphatic $\mathrm{C}-\mathrm{H}$ ), 1653 (amide $\mathrm{C}=\mathrm{O}$ ), 1417 ( $\mathrm{C}=\mathrm{N}$ and $\mathrm{C}=\mathrm{C}$ ), 1217 $(\mathrm{C}=\mathrm{S}), 1001$ (C-N of piperidine), 758 (out-of-plane $\mathrm{C}-\mathrm{H}$ bending); ${ }^{1} \mathrm{H}-\mathrm{NMR}$ (300 MHz, DMSO- $d_{6} ; \delta$, ppm): $1.64\left(6 \mathrm{H}\right.$, s, piperidine $\left.-\mathrm{CH}_{2}\right), 3.96$ and $4.25\left(4 \mathrm{H}\right.$, two s, piperidine $\left.\mathrm{CH}_{2}-\mathrm{N}-\mathrm{CH}_{2}\right), 4.62(2 \mathrm{H}$, $\left.\mathrm{s}, \mathrm{COCH}_{2}\right), 7.57-7.61(2 \mathrm{H}, \mathrm{t}, J=6.6 \mathrm{~Hz}, \mathrm{Ar}-\mathrm{H}), 7.82-7.86(2 \mathrm{H}, \mathrm{t}, J=6.9 \mathrm{~Hz}, \mathrm{Ar}-\mathrm{H}), 8.14-8.16(2 \mathrm{H}$, $\mathrm{d}, J=8.3 \mathrm{~Hz}, \mathrm{Ar}-\mathrm{H}), 8.26-8.29(2 \mathrm{H}, \mathrm{d}, J=8.5 \mathrm{~Hz}, \mathrm{Ar}-\mathrm{H}), 11.14(1 \mathrm{H}, \mathrm{s},-\mathrm{NH}-) ;{ }^{13} \mathrm{C}-\mathrm{NMR}(75 \mathrm{MHz}$, DMSO- $\left.d_{6} ; \delta, \mathrm{ppm}\right): 23.9\left(\mathrm{CH}_{2}\right), 26.0\left(\mathrm{CH}_{2}\right), 51.7\left(\mathrm{CH}_{2}\right), 53.1\left(\mathrm{CH}_{2}\right), 123.2(\mathrm{C}), 125.4(\mathrm{CH}), 126.2(\mathrm{CH})$, 129.6 (CH), 130.9(CH), 140.7 (C), 149.4 (C-9 in 9-aminoacridine), $167.5(\mathrm{C}=\mathrm{O}), 193.7(\mathrm{C}=\mathrm{S})$; HRMS $(\mathrm{m} / \mathrm{z})$ : $[\mathrm{M}+\mathrm{H}]^{+}$calculated for $\mathrm{C}_{21} \mathrm{H}_{21} \mathrm{~N}_{3} \mathrm{OS}_{2}$ : 396.1199, found 396.1184.

2-(9-Acridinylamino)-2-oxoethyl 2-methylpiperidine-1-carbodithioate (4t). Yield: 93.3\%; m.p.: $265.4{ }^{\circ} \mathrm{C}$; FTIR (ATR, $\mathrm{cm}^{-1}$ ): 3255 (amide N-H), 2924 (aliphatic $\mathrm{C}-\mathrm{H}$ ), 1653 (amide $\mathrm{C}=\mathrm{O}$ ), 1417-1463 (C=N and $\mathrm{C}=\mathrm{C}$ ), 1215-1263 (C=S), 1001 (C-N of piperidine), 758 (out-of-plane $\mathrm{C}-\mathrm{H}$ bending); ${ }^{1} \mathrm{H}-\mathrm{NMR}$ (300 MHz, DMSO- $\left.d_{6} ; \delta, \mathrm{ppm}\right): 1.19-1.25\left(9 \mathrm{H}, \mathrm{m}\right.$, piperidine $\left.-\mathrm{CH}_{2},-\mathrm{CH}_{3}\right), 4.26-4.30\left(3 \mathrm{H}, \mathrm{m}\right.$, piperidine $-\mathrm{CH}_{2}$, $-\mathrm{CH}-), 4.61\left(2 \mathrm{H}, \mathrm{s}, \mathrm{COCH}_{2}\right), 7.60(2 \mathrm{H}, \mathrm{t}, J=7.5 \mathrm{~Hz}, \mathrm{Ar}-\mathrm{H}), 7.85(2 \mathrm{H}, \mathrm{t}, J=7.5 \mathrm{~Hz}, \mathrm{Ar}-\mathrm{H}), 8.16(2 \mathrm{H}, \mathrm{d}$, $J=8.6 \mathrm{~Hz}, \mathrm{Ar}-\mathrm{H}), 8.28 \overline{(2 \mathrm{H}}, \mathrm{d}, J=8.7 \mathrm{~Hz}, \mathrm{Ar}-\mathrm{H}), 10.94(1 \mathrm{H}, \mathrm{s},-\mathrm{NH}-) ;{ }^{13} \mathrm{C}-\mathrm{NMR}\left(75 \mathrm{MHz}, \mathrm{DMSO}-d_{6}\right.$;

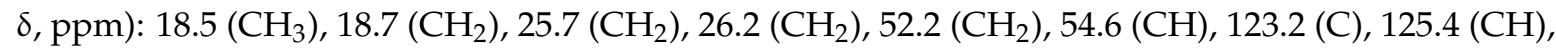
126.2 (CH), $129.6(\mathrm{CH}), 130.9(\mathrm{CH}), 140.3(\mathrm{C}), 149.4$ (C-9 in 9-aminoacridine), $167.6(\mathrm{C}=\mathrm{O}), 194.3(\mathrm{C}=\mathrm{S})$; HRMS $(m / z):[\mathrm{M}+\mathrm{H}]^{+}$calculated for $\mathrm{C}_{22} \mathrm{H}_{23} \mathrm{~N}_{3} \mathrm{OS}_{2}$ : 410.1355, found 410.1338. 
2-(9-Acridinylamino)-2-Oxoethyl 4-Benzylpiperidine-1-Carbodithioate (4u). Yield: 90.7\%; m.p.: $265.4{ }^{\circ} \mathrm{C}$; FTIR (ATR, $\mathrm{cm}^{-1}$ ): 3261 (amide N-H), 2852-2922 (aliphatic C-H), 1653 (amide C=O), 1452-1570 $(\mathrm{C}=\mathrm{N}$ and $\mathrm{C}=\mathrm{C}), 1261(\mathrm{C}=\mathrm{S}), 1016$ ( $\mathrm{C}-\mathrm{N}$ of piperidine), 758 (out-of-plane $\mathrm{C}-\mathrm{H}$ bending); ${ }^{1} \mathrm{H}-\mathrm{NMR}$ (300 MHz, DMSO- $\left.d_{6} ; \delta, \mathrm{ppm}\right): 1.22-1.26\left(5 \mathrm{H}, \mathrm{m}\right.$, piperidine $\left.-\mathrm{CH}_{2},-\mathrm{CH}\right), 3.04-3.08(2 \mathrm{H}, \mathrm{m}$, piperidine $\left.-\mathrm{CH}_{2}-\right), 3.20-3.25\left(2 \mathrm{H}, \mathrm{m}\right.$, piperidine $\left.-\mathrm{CH}_{2}-\right), 3.97\left(2 \mathrm{H}, \mathrm{s}, \mathrm{C}_{6} \mathrm{H}_{5}-\mathrm{CH}_{2}-\right), 4.61\left(2 \mathrm{H}, \mathrm{s}, \mathrm{COCH}_{2}\right), 7.58(2 \mathrm{H}, \mathrm{t}$, $J=7.6 \mathrm{~Hz}, \mathrm{Ar}-\mathrm{H}), 7.67(2 \mathrm{H}, \mathrm{t}, J=7.5 \mathrm{~Hz}, \mathrm{Ar}-\mathrm{H}), 7.77-7.86(5 \mathrm{H}, \mathrm{m}$, benzyl $-\mathrm{CH}), 8.15(2 \mathrm{H}, \mathrm{d}, J=8.7 \mathrm{~Hz}$, $\mathrm{Ar}-\mathrm{H}), 8.28(2 \mathrm{H}, \mathrm{d}, J=8.7 \mathrm{~Hz}, \mathrm{Ar}-\mathrm{H}) ;{ }^{13} \mathrm{C}-\mathrm{NMR}\left(75 \mathrm{MHz}, \mathrm{DMSO}-\mathrm{d}_{6} ; \delta, \mathrm{ppm}\right): 37.1(\mathrm{CH}), 42.0\left(\mathrm{CH}_{2}\right)$, $48.7\left(\mathrm{CH}_{2}\right), 50.6\left(\mathrm{CH}_{2}\right), 54.6\left(\mathrm{CH}_{2}\right), 122.4(\mathrm{C}), 125.5(\mathrm{CH}), 126.0(\mathrm{CH}), 126.3(\mathrm{CH}), 128.7(\mathrm{CH}), 129.5(\mathrm{CH})$, 130.3 (CH), $130.9(\mathrm{CH}), 132.2(\mathrm{CH}), 140.3(\mathrm{C}), 149.2$ (C-9 in 9-aminoacridine), $167.6(\mathrm{C}=\mathrm{O}), 193.9(\mathrm{C}=\mathrm{S})$; HRMS ( $m / z):[\mathrm{M}+\mathrm{H}]^{+}$calculated for $\mathrm{C}_{28} \mathrm{H}_{27} \mathrm{~N}_{3} \mathrm{OS}_{2}: 486.1668$, found 486.1654 .

\subsection{AChE/BChE Activity Assay}

All enzymes were obtained from Sigma-Aldrich (Steinheim, Germany). Ellman's reagent and the substrates were obtained from Fluka (Buchs, Switzerland). The chemicals used to prepare the buffer solution were purchased from Merck (Darmstadt, Germany).

The enzyme inhibitory activities of the synthesized compounds were investigated by applying Ellman's method [35]. Enzyme solutions were dissolved in gelatin solution (1\%; 2.5 units $/ \mathrm{mL})$. Compounds $4 \mathbf{a}-\mathbf{4 u}$ and reference agents were prepared in $2 \%$ DMSO at concentrations of $10^{-3} \mathrm{M}$ and $10^{-4} \mathrm{M}$. The enzyme solution $(20 \mu \mathrm{L} /$ well $)$ and inhibitor solution $(20 \mu \mathrm{L} /$ well $)$ were mixed with buffer $(140 \mu \mathrm{L} /$ well, $\mathrm{pH} 8 \pm 0.1)$ and incubated at $25{ }^{\circ} \mathrm{C}$ for $5 \mathrm{~min}$. Then, the reaction was initiated by the addition of Ellman's reagent 5,5'-Dithiobis(2-nitrobenzoic acid) (DTNB; $20 \mu \mathrm{L} /$ well, $10 \mathrm{mM})$ and the substrate $(10 \mu \mathrm{L} /$ well, $75 \mathrm{mM})$. The absorbance was measured for $10 \mathrm{~min}$ at $412 \mathrm{~nm}$. The enzyme solution was processed as a control. All readings were adjusted with blank-reading. All assays were performed in four independent wells. The same procedure was applied for further concentrations $\left(10^{-5}-10^{-9} \mathrm{M}\right)$ of reference agents and synthesized compounds displaying $\geq 50 \%$ inhibition at initial concentrations $\left(10^{-3}\right.$ and $\left.10^{-4} \mathrm{M}\right)$. The $\mathrm{IC}_{50}$ values were calculated by applying regression analyses using Microsoft Excel 2013 [36]. Absorbance differences between the two readings were taken, and percentage inhibition rates were calculated according to the following formula:

$$
\% \text { Inhibition }=\frac{[(A(C)-A(B))-(A(I)-A(B))]}{(A(C)-A(B))} \times 100 .
$$

Blank (B): the well in which the inhibitor compound and substrate were not added;

Control $(C)$ : the well where the inhibitor compound was not added;

$A(B)$ : the difference in absorbance reading for the blank;

$A(C)$ : the difference in absorbance reading for the control;

$A(I)$ : the difference in absorbance reading for the inhibitor compounds.

\subsection{Enzyme Kinetics}

In the kinetics studies, the assay protocol specified for the inhibition assay was identical. However, unlike the inhibition method, the concentrations of the most active inhibitor compound, $4 \mathbf{n}$, were used at the calculated $\mathrm{IC}_{50} / 2, \mathrm{IC}_{50}$, and $2 \times \mathrm{IC}_{50}$ values. The substrate $\mathrm{BTCI}$ solution with 10 serial dilutions at different concentrations in the range of $150-0.2929 \mathrm{mM}$ were prepared and used. Measurements were performed one of two ways, in the presence and in the absence of the inhibitor. Firstly, the solutions of compound $4 \mathrm{n}$ at three different concentrations were added to the wells $(20 \mu \mathrm{L} /$ well). After addition of $\mathrm{BChE}(20 \mu \mathrm{L} /$ well), the mixture was incubated under the same conditions as the activity assay. Then, the reaction was initiated by the addition of Ellman's reagent (DTNB; $20 \mu \mathrm{L} /$ well, $10 \mathrm{mM}$ ) and the substrate $(10 \mu \mathrm{L} /$ well, $75 \mathrm{mM})$. The absorbance was measured for $10 \mathrm{~min}$ at $412 \mathrm{~nm}$. The enzyme solution was processed as a control. All readings were adjusted with blank-reading. The results were analyzed as Lineweaver-Burk plots using Microsoft Office Excel 2013 [15]. 


\subsection{Molecular Docking}

The docking study was used to discover the binding modes of the most active compound, $\mathbf{4 n}$, to the BChE active site. The structure of Homo sapiens BChE (PDB ID: 4BDS) [32], including tacrine in the active site, was retrieved from the Protein Data Bank server (www.pdb.org). The structure of $4 \mathbf{n}$ was assembled with the Schrödinger Maestro (Schrödinger, 2016) interface [37], and was then directed to the Protein Preparation Wizard protocol of the Schrödinger Suite 2016 Update 2 [38]. The LigPrep 3.8 [39] software was used to accurately prepare compound $\mathbf{4 n}$ for the assignment of protonation regions at $\mathrm{pH} 8.0 \pm 1.0$ and the atom types. Bond orders and hydrogen atoms in the structure were fixed as required. The Glide 7.1 program [40] was used to generate the grid, and docking analyses were carried out using the single-precision docking mode (SP).

\subsection{Cell Viability Assay and Selectivity Indexes}

Healthy murine embryonic fibroblast cells (NIH3T3 cell line) were used for the cytotoxicity tests. The cell line and selected compounds were prepared for an MTT assay as reported previously [41]. The optical densities (OD) of 96 well plates were recorded at $540 \mathrm{~nm}$. The percentage inhibition was determined for each concentration according to the formula below, and the $\mathrm{IC}_{50}$ values were calculated by applying regression analyses using Microsoft Excel 2013 [15].

$$
\% \text { Inhibition }=100-\frac{(\text { OD of sample })}{(\text { OD of solvent })} \times 100 .
$$

\subsection{BBB Permeability and Drug-Likeness Score (DLS)}

With a specific end goal of assessing some important biological parameters for the active compounds (4a, 4b, 4e, 4i, 4m, 4n, 4o, and $4 \mathrm{t})$, BBB permeability and DLS were predicted using an online BBB Predictor and Molsoft's chemical fingerprints mode, respectively [42,43].

\section{Conclusions}

Due to the complicated nature of $\mathrm{AD}$, scientists are still trying to discover a medication to cure this disease. One possible way of overcoming this disease is through the discovery of new compounds that may be useful in its treatment. Hence, 21 new derivatives of 2-(9-acridinylamino)-2-oxoethyl-piperazinyl/piperidinyl/morpholinylcarbodithioate (4a-4u) were designed, synthesized, and screened for their inhibition potential toward ChEs. Eight derivatives (4a, $4 \mathbf{b}, 4 \mathrm{e}, 4 \mathrm{i}, 4 \mathrm{~m}, 4 \mathrm{n}, 4 \mathbf{o}$, and $4 \mathrm{t}$ ) demonstrated a specific and promising action against $\mathrm{BChE}$, from which only one compound (4n) was able to inhibit both enzymes. BChE kinetics and molecular modeling studies were applied to investigate the binding mode of the lead compound, $\mathbf{4 n}$, to the enzyme's active site. Moreover, the result of cytotoxic activity against NIH3T3 cells indicated that the active derivatives showed no cytotoxicity, suggesting a safety profile of these compounds. Similarly, the BBB permeability and DLS studies indicated the relative success of the most active derivatives.

Supplementary Materials: The following are available online, Table S1: Inhibitory Activity (\%) of the compounds $\mathbf{4 a}-\mathbf{4 u}$ against $\mathrm{AChE}$ and $\mathrm{BChE}$., Table S2: Inhibitory activity (\%) and $\mathrm{IC}_{50}$ values of the selected compounds against AChE and BChE.

Author Contributions: Y.Ö. and Z.A.K. conceived and designed the experiments; W.H. performed the synthesis; S.L. performed the analysis; B.N.S. performed the activity tests; B.N.S. performed the docking studies; B.K. and S.I. performed the toxicity tests; W.H., B.N.S., S.L., B.K., S.I., Y.Ö., and Z.A.K. wrote the paper.

Funding: This study was financially supported by the Anadolu University Scientific Projects Fund, Project No: 1805 S191.

Conflicts of Interest: The authors declare no conflict of interest. 


\section{References}

1. World Alzheimer Report 2015. Available online: https://www.alz.co.uk/research/worldalzheimerreport2 015summary.pdf (accessed on 26 January 2017).

2. Dementia: A Public Health Priority. Available online: http://www.who.int/mental_health/publications/d ementia_report_2012/en/ (accessed on 26 January 2017).

3. Melnikova, I. Therapies for Alzheimer's disease. Nat. Rev. Drug Discov. 2007, 6, 341-342. [CrossRef] [PubMed]

4. Gauthier, S. Advances in the pharmacotherapy of Alzheimer's disease. Can. Med. Assoc. J. 2002, 166, 616-623.

5. Darvesh, S.; Grantham, D.L.; Hopkins, D.A. Distribution of butyrylcholinesterase in the human amygdala and hippocampal formation. J. Comp. Neurol. 1998, 393, 374-390. [CrossRef]

6. Decker, M.; Kraus, B.; Heilmann, J. Design, synthesis and pharmacological evaluation of hybrid molecules out of quinazolinimines and lipoic acid lead to highly potent and selective butyrylcholinesterase inhibitors with antioxidant properties. Bioorg. Med. Chem. 2008, 16, 4252-4261. [CrossRef] [PubMed]

7. Carolan, C.G.; Dillon, G.P.; Khan, D.; Ryder, S.A.; Gaynor, J.M.; Reidy, S.; Marquez, J.F.; Jones, M.; Holland, V.; Gilmer, J.F. Isosorbide-2-benzyl carbamate-5-salicylate, a peripheral anionic site binding subnanomolar selective butyrylcholinesterase inhibitor. J. Med. Chem. 2010, 53, 1190-1199. [CrossRef] [PubMed]

8. Nawaz, S.A.; Ayaz, M.; Brandt, W.; Wessjohann, L.A.; Westermann, B. Cation- $\pi$ and $\pi-\pi$ stacking interactions allow selective inhibition of butyrylcholinesterase by modified quinine and cinchonidine alkaloids. Biochem. Biophys. Res. Commun. 2011, 404, 935-940. [CrossRef] [PubMed]

9. Guillozet, A.L.; Smiley, J.F.; Mash, D.C.; Mesulam, M.M. Butyrylcholinesterase in the life cycle of amyloid plaques. Ann. Neurol. 1997, 42, 909-918. [CrossRef] [PubMed]

10. Podoly, E.; Shalev, D.E.; Shenhar-Tsarfaty, S.; Bennett, E.R.; Ben Assayag, E.; Wilgus, H.; Livnah, O.; Soreq, H. The butyrylcholinesterase K variant confers structurally derived risks for Alzheimer pathology. J. Biol. Chem. 2009, 284, 17170-17179. [CrossRef] [PubMed]

11. Shen, Z.X. Future perspectives of AD research and clinical practice. Med. Hypotheses 2004, 63, $298-307$. [CrossRef] [PubMed]

12. Lemke, T.L.; Williams, D.A.; Roche, V.F.; Zito, S.W. Foye's Principles of Medicinal Chemistry, 6th ed.; Lippincott Williams \& Wilkins: Philadelphia, PA, USA, 2008.

13. Madalageri, P.M.; Kotresh, O. Synthesis, DNA protection and antimicrobial activity of some novel chloromethyl benzimidazole derivatives bearing dithiocarbamates. J. Chem. Pharm. Res. 2012, 4, 2697-2703.

14. Levent, S.; Acar-Çevik, U.A.; Sağlık, B.N.; Özkay, Y.; Can, O.D.; Özkay, U.D.; Uçucu, Ü. Anticholinesterase activity screening of some novel dithiocarbamate derivatives including piperidine and piperazine moieties. Phosphorus Sulfur Silicon Relat. Elem. 2017, 192, 469-474. [CrossRef]

15. Demir Özkay, Ü.; Can, Ö.D.; Sağlık, B.N.; Acar Çevik, U.; Levent, S.; Özkay, Y.; Ilgın, S.; Atlı, Ö. Design, synthesis, and AChE inhibitory activity of new benzothiazole-piperazines. Bioorg. Med. Chem. Lett. 2016, 26, 5387-5394. [CrossRef] [PubMed]

16. Korabecny, J.; Andrs, M.; Nepovimova, E.; Dolezal, R.; Babkova, K.; Horova, A.; Malinak, D.; Mezeiova, E.; Gorecki, L.; Sepsova, V.; et al. 7-Methoxytacrine- $p$-anisidine hybrids as novel dual binding site acetylcholinesterase inhibitors for Alzheimer's disease treatment. Molecules 2015, 20, 22084-22101. [CrossRef] [PubMed]

17. Khoury, R.; Patel, K.; Gold, J.; Hinds, S.; Grossberg, G.T. Recent progress in the pharmacotherapy of Alzheimer's disease. Drugs Aging 2017, 34, 811-820. [CrossRef] [PubMed]

18. Korabecny, J.; Musilek, K.; Holas, O.; Nepovimova, E.; Jun, D.; Zemek, F.; Opletalova, V.; Patocka, J.; Dohnal, V.; Nachon, F.; et al. Synthesis and in vitro evaluation of $\mathrm{N}$-(bromobut-3-en-2-yl)-7-methoxy-1,2,3,4-tetrahydroacridin-9-amine as a cholinesterase inhibitor with regard to Alzheimer's disease treatment. Molecules 2010, 15, 8804-8812. [CrossRef] [PubMed]

19. Panek, D.; Więckowska, A.; Wichur, T.; Bajda, M.; Godyń, J.; Jończyk, J.; Mika, K.; Janockova, J.; Soukup, O.; Knez, D.; et al. Design, synthesis and biological evaluation of new phthalimide and saccharin derivatives with alicyclic amines targeting cholinesterases, beta-secretase and amyloid beta aggregation. Eur. J. Med. Chem. 2017, 125, 676-695. [CrossRef] [PubMed] 
20. Wu, W.Y.; Dai, Y.C.; Li, N.G.; Dong, Z.X.; Gu, T.; Shi, Z.H.; Xue, X.; Tang, Y.P.; Duan, J.A. Novel multitarget-directed tacrine derivatives as potential candidates for the treatment of Alzheimer's disease. J. Enzyme Inhib. Med. Chem. 2017, 32, 572-587. [CrossRef] [PubMed]

21. Spilovska, K.; Korabecny, J.; Sepsova, V.; Jun, D.; Hrabinova, M.; Jost, P.; Muckova, L.; Soukup, O.; Janockova, J.; Kucera, T.; et al. Novel tacrine-scutellarin hybrids as multipotent anti-Alzheimer's agents: Design, synthesis and biological evaluation. Molecules 2017, 22, 1006. [CrossRef] [PubMed]

22. Košak, U.; Brus, B.; Knez, D.; Šink, R.; Žakelj, S.; Trontelj, J.; Pišlar, A.; Šlenc, J.; Gobec, M.; Živin, M.; et al. Development of an in vivo active reversible butyrylcholinesterase inhibitor. Sci. Rep. 2016, 6, 39495. [CrossRef] [PubMed]

23. Sawatzky, E.; Wehle, S.; Kling, B.; Wendrich, J.; Bringmann, G.; Sotriffer, C.A.; Heilmann, J.; Decker, M. Discovery of highly selective and nanomolar carbamate-based butyrylcholinesterase inhibitors by rational investigation into their inhibition mode. J. Med. Chem. 2016, 59, 2067-2082. [CrossRef] [PubMed]

24. Krátký, M.; Štěpánková, S.; Vorčáková, K.; Švarcová, M.; Vinsova, J. Novel cholinesterase inhibitors based on $o$-aromatic $N, N$-disubstituted carbamates and thiocarbamates. Molecules 2016, 21, 191. [CrossRef] [PubMed]

25. Nicolet, Y.; Lockridge, O.; Masson, P.; Fontecilla-Camps, J.C.; Nachon, F. Crystal structure of human butyrylcholinesterase and of its complexes with substrate and products. J. Biol. Chem. 2003, 278, 41141-41147. [CrossRef] [PubMed]

26. Lucier, G.W.; McDaniel, O.S.; Matthews, H.B. Microsomal rat liver UDP glucuronyltransferase. Effects of piperonyl butoxide and other factors on enzyme activity. Arch. Biochem. Biophys. 1971, 145, 520-530. [CrossRef]

27. Schneider, R.W. Effects of nonpathogenic strains of fusarium oxysporum on celery root infection by F. oxysporum f. sp. apii and a novel use of the Limeweaver-Burk double reciprocal plot technique. Phytopathology 1984, 74, 646. [CrossRef]

28. Ahmed, M.; Rocha, J.B.; Correa, M.; Mazzanti, C.M.; Zanin, R.F.; Morsch, A.L.; Morsch, V.M.; Schetinger, M.R. Inhibition of two different cholinesterases by tacrine. Chem. Biol. Interact. 2006, 162, 165-171. [CrossRef] [PubMed]

29. Wang, B.; Mai, Y.C.; Li, Y.; Hou, J.Q.; Huang, S.L.; Ou, T.M.; Tan, J.H.; An, L.K.; Li, D.; Gu, L.Q.; et al. Synthesis and evaluation of novel rutaecarpine derivatives and related alkaloids derivatives as selective acetylcholinesterase inhibitors. Eur. J. Med. Chem. 2010, 45, 1415-1423. [CrossRef] [PubMed]

30. Li, Z.; Mu, C.; Wang, B.; Jin, J. Graveoline analogs exhibiting selective acetylcholinesterase inhibitory activity as potential lead compounds for the treatment of alzheimer's disease. Molecules 2016, 21, 132. [CrossRef] [PubMed]

31. Nachon, F.; Carletti, E.; Ronco, C.; Trovaslet, M.; Nicolet, Y.; Jean, L.; Renard, P.Y. Crystal structures of human cholinesterases in complex with huprine $\mathrm{W}$ and tacrine: Elements of specificity for anti-Alzheimer's drugs targeting acetyl- and butyryl-cholinesterase. Biochem. J. 2013, 453, 393-399. [CrossRef] [PubMed]

32. Goodwin, J.T.; Clark, D.E. In Silico Predictions of Blood-Brain Barrier Penetration: Considerations to "Keep in Mind". J. Pharmacol. Exp. Ther. 2005, 315, 477-483. [CrossRef] [PubMed]

33. Wang, X.; Bhatia, P.A.; Daanen, J.F.; Latsaw, S.P.; Rohde, J.; Kolasa, T.; Hakeem, A.A.; Matulenko, M.A.; Nakane, M.; Uchic, M.E.; et al. Synthesis and evaluation of 3-aryl piperidine analogs as potent and efficacious dopamine D4 receptor agonists. Bioorg. Med. Chem. 2005, 13, 4667-4678. [CrossRef] [PubMed]

34. Demir-Özkay, U.; Özkay, Y.; Can, Ö. Synthesis and analgesic effects of 2-(2-carboxyphenylsulfanyl)-N -(4-substitutedphenyl)acetamide derivatives. Med. Chem. Res. 2011, 20, 152-157. [CrossRef]

35. Ellman, G.L.; Courtney, K.D.; Andres, V.J.; Feather-Stone, R.M. A new and rapid colorimetric determination of acetylcholinesterase activity. Biochem. Pharmacol. 1961, 7, 88-95. [CrossRef]

36. Yurttaş, L.; Kaplancıklı, Z.A.; Ozkay, Y. Design, synthesis and evaluation of new thiazole-piperazines as acetylcholinesterase inhibitors. J. Enzyme Inhib. Med. Chem. 2013, 28, 1040-1047. [CrossRef] [PubMed]

37. Maestro, version 10.6; Schrödinger, LLC: New York, NY, USA, 2016.

38. Schrödinger, version 2016-2; Schrödinger, LLC: New York, NY, USA, 2016.

39. LigPrep, version 3.8; Schrödinger, LLC: New York, NY, USA, 2016.

40. Glide, version 7.1; Schrödinger, LLC: New York, NY, USA, 2016.

41. Berridge, M.V.; Herst, P.M.; Tan, A.S. Tetrazolium dyes as tools in cell biology: New insights into their cellular reduction. Biotechnol. Annu. Rev. 2005, 11, 127-152. [PubMed] 
42. The Blood-Brain Barrier (BBB) Prediction Server-CBLigand. Available online: http://www.cbligand.org/B BB/index.php (accessed on 27 February 2017).

43. Drug-Likeness and Molecular Property Prediction. Available online: http://molsoft.com/mprop/ (accessed on 2 March 2017).

Sample Availability: Samples of the compounds $\mathbf{4 a}-\mathbf{4} \mathbf{u}$ are available from the authors.

(C) 2018 by the authors. Licensee MDPI, Basel, Switzerland. This article is an open access article distributed under the terms and conditions of the Creative Commons Attribution (CC BY) license (http://creativecommons.org/licenses/by/4.0/). 